Supporting Information

\title{
Hydrocarbon Dew Point Measurement and Models Evaluation of Synthetic and Real Natural Gases
}

Pu Zhang ${ }^{1,2, *}$, Li Zhou ${ }^{1,2}$, Wenping Zeng ${ }^{1,2}$, Gang Xiong ${ }^{1,2}$, Hongfa Huang ${ }^{1,2}$, Li Cai ${ }^{1,2}$,

Huiyan $Y e^{1}$ and Shanshan $Q u^{1,2}$

${ }^{1}$ Research Institute of Natural Gas Technology, PetroChina Southwest Oil and Gasfield Company, China National Petroleum Corporation(CNPC), Chengdu, Sichuan, China

${ }^{2}$ Key Laboratory of Natural Gas Quality and Energy Measurement, CNPC, Chengdu, Sichuan, China 
Table S1. Natural gas composition of 28 SNGs (SNG1 SNG25 are collected from other literatures)

\begin{tabular}{|c|c|c|c|c|c|c|c|c|c|c|c|c|c|c|}
\hline & $\mathrm{SNG}^{33}$ & $\mathrm{SNG}^{33}$ & $\mathrm{SNG}^{33}$ & $\mathrm{SNG}^{33}$ & $\mathrm{SNG}^{33}$ & SNG $6^{10}$ & $\mathrm{SNG7}^{10}$ & SNG8 ${ }^{10}$ & SNG9 $9^{10}$ & SNG10 10 & $\mathrm{SNG}^{10}{ }^{10}$ & $\mathrm{SNG}_{12}{ }^{4}$ & $\mathrm{SNG} 3^{4}$ & SNG14 ${ }^{4}$ \\
\hline $\mathrm{N}_{2}$ & - & - & - & - & - & 0.3481 & - & - & - & - & - & 0.6180 & 0.3130 & 2.8000 \\
\hline $\mathrm{CO}_{2}$ & - & - & - & - & - & - & - & - & - & - & - & 0.1870 & 0.2020 & 0.2000 \\
\hline $\mathrm{C}_{1}$ & 93.5050 & 84.2800 & 96.6110 & 94.0850 & 93.6000 & 93.0413 & 93.1210 & 93.1857 & 83.9403 & 84.0795 & 84.1137 & 98.9430 & 90.4183 & 96.6159 \\
\hline $\mathrm{C}_{2} \mathrm{H}_{6}$ & 2.9720 & 10.0670 & 0.0000 & 4.4680 & 2.6300 & 2.9959 & 3.0483 & 3.0642 & 10.0159 & 9.9730 & 9.9574 & 0.0820 & 8.0380 & 0.1800 \\
\hline $\mathrm{C}_{3} \mathrm{H}_{8}$ & 1.0080 & 4.0280 & 0.0000 & 0.0000 & 0.0000 & 0.9649 & 0.9936 & 1.0139 & 4.1088 & 4.0365 & 4.1112 & 0.0650 & 0.8010 & 0.1029 \\
\hline $\mathrm{i}-\mathrm{C}_{4}$ & 1.0500 & 0.5970 & 1.5270 & 0.0000 & 1.4900 & 1.0038 & 1.0323 & 1.0266 & 0.6012 & 0.6029 & 0.5762 & 0.0500 & 0.0810 & 0.0499 \\
\hline $\mathrm{n}-\mathrm{C}_{4}$ & 1.4650 & 1.0280 & 1.4750 & 0.0000 & 1.4900 & 1.3482 & 1.5099 & 1.5214 & 1.0314 & 1.0122 & 1.0306 & & 0.1230 & 0.0095 \\
\hline \multicolumn{15}{|l|}{ neoC $_{5}$} \\
\hline $\mathrm{i}-\mathrm{C}_{5}$ & - & - & - & - & - & - & - & - & - & - & 0.0028 & 0.0170 & 0.0100 & 0.0166 \\
\hline$n-C_{5}$ & - & - & 0.3850 & 1.4470 & 0.7950 & - & - & - & - & - & 0.0078 & & 0.0079 & \\
\hline$n-C_{6}$ & - & - & - & - & - & - & - & - & - & - & 0.0050 & 0.0320 & 0.0047 & 0.0160 \\
\hline $\mathrm{n}-\mathrm{C}_{7}$ & - & - & - & - & - & - & - & 0.1982 & - & - & 0.1954 & 0.0027 & 0.0011 & 0.0054 \\
\hline cyclohexane & - & - & - & - & - & 0.2978 & - & - & 0.3025 & 0.2959 & - & - & - & - \\
\hline benzene & - & - & - & - & - & - & 0.2948 & - & - & - & - & - & - & - \\
\hline$n-C_{8}$ & - & - & - & - & - & - & - & - & - & - & - & 0.0033 & & 0.0038 \\
\hline
\end{tabular}




\begin{tabular}{|c|c|c|c|c|c|c|c|c|c|c|c|c|c|c|}
\hline & $\mathrm{SNG} 5^{4}$ & SNG16 ${ }^{4}$ & SNG17 ${ }^{5}$ & SNG18 & SNG19 ${ }^{5}$ & SNG $20^{5}$ & SNG $21^{7}$ & $\mathrm{SNG}_{22^{7}}$ & $\mathrm{SNG} 3^{7}$ & SNG $24^{7}$ & SNG25 ${ }^{11}$ & SNG26 & SNG27 & SNG28 \\
\hline $\mathrm{N}_{2}$ & 6.9000 & 5.6510 & 0.6700 & 0.4800 & 0.8620 & 0.4100 & 1.5590 & 0.7720 & - & - & 5.6700 & 0.5096 & 0.7056 & 0.7772 \\
\hline $\mathrm{CO}_{2}$ & 0.5100 & 0.2840 & - & - & - & - & 25.9080 & 1.7000 & - & - & 0.2870 & 0.1500 & 0.2940 & 0.0549 \\
\hline $\mathrm{CH}_{4}$ & 88.1882 & 83.3482 & 89.9584 & 88.7634 & 86.4838 & 96.4654 & 69.1140 & 84.4460 & 89.0000 & 99.9000 & 83.3000 & 92.9111 & 85.5114 & 89.7150 \\
\hline $\mathrm{C}_{2} \mathrm{H}_{6}$ & 2.7200 & 7.5260 & 8.2200 & 8.5400 & 9.8320 & 2.5100 & 2.6200 & 8.6830 & 7.0000 & - & 7.5600 & 4.8700 & 8.8100 & 6.1800 \\
\hline $\mathrm{C}_{3} \mathrm{H}_{8}$ & 0.8500 & 2.0090 & 0.9000 & 1.6800 & 2.3880 & 0.2130 & 0.4230 & 3.2970 & - & - & 2.0200 & 0.9350 & 2.9400 & 1.9100 \\
\hline $\mathrm{i}-\mathrm{C}_{4}$ & 0.1700 & 0.3050 & 0.1100 & 0.2200 & 0.1830 & 0.1840 & 0.1050 & 0.2930 & - & - & 0.3100 & 0.2410 & 0.6060 & 0.3480 \\
\hline $\mathrm{n}-\mathrm{C}_{4}$ & 0.3200 & 0.5200 & 0.1300 & 0.2900 & 0.2310 & 0.1970 & 0.1040 & 0.5890 & 4.0000 & - & 0.5260 & 0.1490 & 0.5820 & 0.4280 \\
\hline neoC $_{5}$ & & & & & & & & & & & & 0.0071 & 0.0060 & 0.0089 \\
\hline $\mathrm{i}-\mathrm{C}_{5}$ & 0.0850 & 0.1200 & 0.0084 & 0.0182 & 0.0139 & 0.0096 & 0.0340 & 0.0840 & - & - & 0.1190 & 0.0814 & 0.2210 & 0.1440 \\
\hline$n-C_{5}$ & 0.0940 & 0.1440 & 0.0032 & 0.0084 & 0.0063 & 0.0100 & 0.0230 & 0.0860 & - & - & 0.1440 & 0.0308 & 0.1120 & 0.1330 \\
\hline $\mathrm{n}-\mathrm{C}_{6}$ & 0.1190 & 0.0680 & - & - & - & 0.0010 & 0.1100 & 0.0500 & - & - & 0.0680 & 0.1150 & 0.2120 & 0.3010 \\
\hline $\mathrm{n}-\mathrm{C}_{7}$ & 0.0258 & 0.0138 & - & - & - & - & - & - & - & 0.1000 & 0.0137 & - & - & - \\
\hline cyclohexane & - & - & - & - & - & - & - & - & - & - & - & - & - & - \\
\hline benzene & - & - & - & - & - & - & - & - & - & - & - & - & - & - \\
\hline $\mathrm{n}-\mathrm{C}_{8}$ & 0.0180 & 0.0110 & - & - & - & - & - & - & - & - & 0.0110 & - & - & - \\
\hline
\end{tabular}


Table S2. Natural gas composition of 14 RGs (SNG1 SNG13 are collected from other literatures)

\begin{tabular}{|c|c|c|c|c|c|c|c|c|c|c|c|c|c|c|}
\hline & $\mathrm{RG} 1^{1}$ & $\mathrm{RG} 2^{1}$ & $\mathrm{RG}^{1}$ & $\mathrm{RG} 4^{1}$ & $\mathrm{RG}^{1}{ }^{1}$ & $\mathrm{RG}^{1}$ & $\mathrm{RG} 7^{8}$ & $\mathrm{RG}^{8}$ & $\mathrm{RG} 9^{8}$ & $\mathrm{RG} 10^{8}$ & $\mathrm{RG}_{1} 1^{8}$ & $\mathrm{RG} 12^{8}$ & $\mathrm{RG}^{1} 3^{11}$ & RG14 \\
\hline $\mathrm{He}$ & - & - & - & - & - & - & - & - & - & - & - & - & - & 0.010900 \\
\hline $\mathrm{H}_{2}$ & - & - & - & - & - & - & - & - & - & - & - & - & - & 0.014800 \\
\hline $\mathrm{N}_{2}$ & 0.624620 & 0.510760 & 0.505520 & 0.622760 & 0.727660 & 0.715190 & 5.693000 & 5.612000 & 4.377000 & 2.224000 & 3.450000 & 3.048000 & 0.550840 & 0.861100 \\
\hline $\mathrm{CO}_{2}$ & 1.699200 & 1.892240 & 1.789140 & 1.671510 & 2.419470 & 2.551010 & 0.205000 & 0.199000 & 0.670000 & 1.047000 & 0.615000 & 1.201000 & 3.576060 & 0.054100 \\
\hline $\mathrm{CH}_{4}$ & 88.390310 & 86.629550 & 85.449760 & 88.166040 & 83.017828 & 82.672538 & 82.770000 & 83.426000 & 83.351000 & 84.033000 & 83.965000 & 82.816000 & 78.989300 & 90.784200 \\
\hline $\mathrm{C}_{2} \mathrm{H}_{6}$ & 5.346310 & 6.190280 & 7.089920 & 5.484760 & 7.792991 & 7.766741 & 7.901000 & 7.675000 & 8.828000 & 9.333000 & 8.654000 & 8.934000 & 9.047690 & 6.449700 \\
\hline $\mathrm{C}_{3} \mathrm{H}_{8}$ & 2.225150 & 2.794060 & 2.930220 & 2.313470 & 3.795250 & 3.901020 & 2.137000 & 1.989000 & 2.038000 & 2.396000 & 2.265000 & 2.831000 & 4.961210 & 1.097300 \\
\hline $\mathrm{i}-\mathrm{C}_{4}$ & 0.386580 & 0.463460 & 0.553470 & 0.363850 & 0.521740 & 0.536630 & 0.344000 & 0.298000 & 0.229000 & 0.338000 & 0.353000 & 0.341000 & 0.683570 & 0.164200 \\
\hline $\mathrm{n}-\mathrm{C}_{4}$ & 0.614030 & 0.752360 & 0.849670 & 0.625200 & 0.980780 & 1.064670 & 0.529000 & 0.453000 & 0.330000 & 0.421000 & 0.446000 & 0.661000 & 1.279200 & 0.205300 \\
\hline neoC $_{5}$ & 0.013410 & 0.016010 & 0.017300 & 0.011290 & 0.025510 & 0.026810 & - & - & - & - & - & - & 0.016800 & 0.004200 \\
\hline $\mathrm{i}-\mathrm{C}_{5}$ & 0.179030 & 0.210940 & 0.236160 & 0.186160 & 0.211280 & 0.227010 & 0.124000 & 0.098000 & 0.055000 & 0.080000 & 0.088000 & 0.057000 & 0.266990 & 0.071000 \\
\hline $\mathrm{n}-\mathrm{C}_{5}$ & 0.160640 & 0.183660 & 0.218100 & 0.174300 & 0.218080 & 0.234100 & 0.138000 & 0.110000 & 0.055000 & 0.060000 & 0.075000 & 0.056000 & 0.283180 & 0.067500 \\
\hline сус- $\mathrm{C}_{5}$ & 0.014800 & 0.016440 & 0.015220 & 0.016650 & 0.013100 & 0.013740 & - & - & - & 0.003000 & 0.004000 & 0.003000 & 0.015140 & - \\
\hline $22 \mathrm{M}-\mathrm{C}_{4}$ & 0.004910 & 0.005530 & 0.008830 & 0.005190 & 0.003050 & 0.003240 & - & - & - & - & - & - & 0.003070 & - \\
\hline $23 \mathrm{M}-\mathrm{C}_{4}$ & 0.008650 & 0.009430 & 0.017190 & 0.008960 & 0.006260 & 0.006620 & - & - & - & - & - & - & 0.007190 & - \\
\hline $2 \mathrm{M}-\mathrm{C}_{5}$ & 0.038130 & 0.040190 & 0.047350 & 0.042100 & 0.041060 & 0.043540 & - & - & - & - & - & - & 0.051690 & - \\
\hline $3 \mathrm{M}-\mathrm{C}_{5}$ & 0.021050 & 0.022110 & 0.024980 & 0.022910 & 0.021280 & 0.022450 & - & - & - & 0.023000 & 0.028000 & 0.019000 & 0.026960 & - \\
\hline $\mathrm{n}-\mathrm{C}_{6}$ & 0.049760 & 0.050260 & 0.053550 & 0.055310 & 0.054000 & 0.056940 & 0.082906 & 0.069857 & 0.030888 & 0.041000 & 0.053000 & 0.038000 & 0.068100 & 0.075600 \\
\hline M-Сус-C 5 & - & - & - & - & - & - & - & - & - & 0.000400 & 0.002100 & 0.000800 & - & - \\
\hline benzene & 0.035580 & 0.036160 & 0.008340 & 0.039930 & 0.016290 & 0.017710 & - & - & - & 0.003400 & 0.005100 & 0.002000 & 0.017640 & 0.023400 \\
\hline $\mathrm{CC}_{6}$ & 0.087130 & 0.086560 & 0.092850 & 0.093230 & 0.056290 & 0.058470 & - & - & - & 0.000200 & 0.002100 & 0.000800 & 0.064460 & 0.011900 \\
\hline
\end{tabular}




\begin{tabular}{|c|c|c|c|c|c|c|c|c|c|c|c|c|c|c|}
\hline & $\mathrm{RG} 1^{1}$ & $\mathrm{RG}^{1}$ & $\mathrm{RG}^{1}$ & $\mathrm{RG} 4^{1}$ & $\mathrm{RG}^{1}$ & $\mathrm{RG}^{1}$ & $\mathrm{RG} 7^{8}$ & $\mathrm{RG}^{8}$ & $\mathrm{RG}^{8}$ & $\mathrm{RG} 10^{8}$ & $\mathrm{RG}_{11^{8}}$ & $\mathrm{RG}_{12}{ }^{8}$ & $\mathrm{RG}^{1}{ }^{11}$ & RG14 \\
\hline $\mathrm{n}-\mathrm{C}_{7}$ & 0.032930 & 0.029740 & 0.039230 & 0.033040 & 0.032440 & 0.033810 & 0.009313 & 0.008754 & 0.003369 & 0.004100 & 0.006500 & 0.002900 & 0.040320 & 0.045000 \\
\hline M-Сус- ${ }_{6}$ & - & - & - & - & - & - & - & - & - & 0.001470 & 0.002170 & 0.001300 & - & 0.025600 \\
\hline toluene & 0.022860 & 0.020880 & 0.004800 & 0.021990 & 0.010230 & 0.010670 & - & - & - & 0.000570 & 0.000790 & 0.000370 & 0.010370 & 0.008700 \\
\hline Сус- ${ }_{7}$ & 0.035600 & 0.031710 & 0.035470 & 0.033880 & 0.024220 & 0.024920 & - & - & - & - & - & - & 0.025070 & - \\
\hline $\mathrm{n}-\mathrm{C}_{8}$ & 0.004950 & 0.003940 & 0.007180 & 0.004080 & 0.005660 & 0.006090 & 0.002263 & 0.001989 & 0.000798 & 0.001040 & 0.001700 & 0.000900 & 0.007940 & 0.019000 \\
\hline Сус-С 8 & 0.000980 & 0.000780 & 0.002420 & 0.000750 & 0.001690 & 0.001920 & - & - & - & - & - & - & 0.001950 & - \\
\hline $\mathrm{n}-\mathrm{C}_{9}$ & 0.000720 & 0.000630 & 0.001640 & 0.000510 & 0.001210 & 0.001320 & 0.002549 & 0.002085 & 0.000918 & 0.001400 & 0.002260 & 0.001020 & 0.002030 & 0.005900 \\
\hline $\mathrm{n}-\mathrm{C}_{10}$ & 0.000000 & 0.000000 & 0.000520 & 0.000000 & 0.000530 & 0.000610 & 0.000091 & 0.000073 & 0.000044 & 0.000052 & 0.000076 & 0.000049 & 0.000900 & 0.000600 \\
\hline m-xylene & 0.002670 & 0.002320 & 0.001170 & 0.002130 & 0.002100 & 0.002230 & - & - & - & - & - & - & 0.002320 & - \\
\hline p-xylene & - & - & - & - & - & - & & & & 0.000130 & 0.000190 & 0.000080 & - & - \\
\hline$n-C_{11}$ & - & - & - & - & - & - & 0.000019 & 0.000014 & 0.000013 & 0.000012 & 0.000016 & 0.000009 & - & - \\
\hline $\mathrm{n}-\mathrm{C}_{12}$ & - & - & - & - & - & - & 0.000009 & 0.000004 & 0.000006 & 0.000006 & 0.000009 & 0.000003 & - & - \\
\hline
\end{tabular}


Table S3. Measured hydrocarbon dew point of 25 SNGs from other literatures

\begin{tabular}{|c|c|c|c|c|c|c|c|c|c|}
\hline $\begin{array}{l}\text { SNG1 } \\
/ \mathrm{MPa}\end{array}$ & $\begin{array}{l}\text { SNG1 } \\
/{ }^{\circ} \mathrm{C}\end{array}$ & $\begin{array}{l}\text { SNG2 } \\
/ \mathrm{MPa}\end{array}$ & $\begin{array}{c}\text { SNG2 } \\
/{ }^{\circ} \mathrm{C}\end{array}$ & $\begin{array}{l}\text { SNG3 } \\
/ \mathrm{MPa}\end{array}$ & $\begin{array}{c}\text { SNG3 } \\
/{ }^{\circ} \mathrm{C}\end{array}$ & $\begin{array}{c}\text { SNG4 } \\
/ \mathrm{MPa}\end{array}$ & $\begin{array}{c}\text { SNG4 } \\
/{ }^{\circ} \mathrm{C}\end{array}$ & $\begin{array}{l}\text { SNG5 } \\
/ \mathrm{MPa}\end{array}$ & $\begin{array}{c}\text { SNG5 } \\
/{ }^{\circ} \mathrm{C}\end{array}$ \\
\hline 8.92 & -26.95 & 8.70 & -16.95 & 7.92 & -9.45 & 9.44 & -7.15 & 9.32 & -4.45 \\
\hline 8.62 & -25.25 & 8.39 & -15.15 & 7.54 & -7.85 & 9.10 & -4.85 & 9.04 & -1.95 \\
\hline 8.13 & -22.55 & 8.09 & -14.15 & 7.06 & -7.05 & 8.66 & -2.95 & 8.52 & -0.75 \\
\hline 7.50 & -19.95 & 7.53 & -12.45 & 6.56 & -6.15 & 8.20 & -1.05 & 8.10 & 1.15 \\
\hline 7.10 & -18.85 & 7.07 & -11.65 & 6.04 & -5.35 & 7.70 & -0.75 & 7.68 & 2.15 \\
\hline 6.61 & -17.75 & 6.16 & -10.95 & 5.56 & -4.85 & 7.22 & 1.25 & 7.16 & 2.95 \\
\hline 6.13 & -17.05 & 5.22 & -11.95 & 5.06 & -4.95 & 6.62 & 1.55 & 6.64 & 4.45 \\
\hline 6.14 & -17.35 & 4.62 & -12.75 & 4.58 & -5.15 & 6.12 & 2.95 & 6.18 & 5.25 \\
\hline 5.60 & -16.85 & 3.78 & -15.25 & 4.10 & -5.75 & 5.66 & 3.65 & 5.64 & 5.15 \\
\hline 5.11 & -16.85 & 3.40 & -16.45 & 3.58 & -6.75 & 4.98 & 3.65 & 5.12 & 5.25 \\
\hline 4.61 & -17.25 & 3.02 & -18.35 & 3.08 & -8.25 & 4.52 & 3.45 & 4.62 & 4.35 \\
\hline 4.11 & -17.95 & 2.50 & -21.35 & 2.60 & -10.35 & 4.06 & 2.75 & 4.08 & 3.45 \\
\hline 3.56 & -17.85 & 1.96 & -25.25 & 2.08 & -13.25 & 3.54 & 1.95 & 3.60 & 2.55 \\
\hline 3.06 & -19.85 & 3.06 & -18.25 & 1.58 & -17.25 & 2.56 & -0.85 & 3.10 & 0.45 \\
\hline 2.60 & -21.75 & 2.54 & -20.95 & 1.06 & -23.45 & 2.04 & -3.55 & 2.60 & -1.85 \\
\hline 2.08 & -25.05 & 2.06 & -24.75 & 0.64 & -31.25 & 1.62 & -6.45 & 2.08 & -4.65 \\
\hline 1.56 & -29.85 & 1.54 & -29.85 & & & 1.54 & -7.25 & 1.56 & -9.05 \\
\hline \multirow[t]{6}{*}{1.04} & -36.55 & 1.04 & -36.95 & & & 1.04 & -12.35 & 1.06 & -15.05 \\
\hline & & & & & & 0.84 & -15.75 & 0.58 & -25.25 \\
\hline & & & & & & 0.68 & -18.55 & 0.34 & -32.35 \\
\hline & & & & & & 0.54 & -22.05 & & \\
\hline & & & & & & 0.42 & -25.55 & & \\
\hline & & & & & & 0.30 & -29.75 & & \\
\hline
\end{tabular}




\begin{tabular}{|c|c|c|c|c|c|c|c|c|c|}
\hline $\begin{array}{l}\text { SNG6 } \\
\text { /MPa }\end{array}$ & $\begin{array}{c}\text { SNG6 } \\
/{ }^{\circ} \mathrm{C}\end{array}$ & $\begin{array}{c}\text { SNG7 } \\
/ \mathrm{MPa}\end{array}$ & $\begin{array}{c}\text { SNG7 } \\
/{ }^{\circ} \mathrm{C}\end{array}$ & $\begin{array}{c}\text { SNG8 } \\
\text { /MPa }\end{array}$ & $\begin{array}{c}\text { SNG8 } \\
/{ }^{\circ} \mathrm{C}\end{array}$ & $\begin{array}{c}\text { SNG9 } \\
\text { /MPa }\end{array}$ & $\begin{array}{c}\text { SNG9 } \\
/{ }^{\circ} \mathrm{C}\end{array}$ & $\begin{array}{c}\text { SNG10 } \\
/ \mathrm{MPa}\end{array}$ & $\begin{array}{c}\text { SNG10 } \\
/{ }^{\circ} \mathrm{C}\end{array}$ \\
\hline 9.88 & -9.05 & 10.22 & -15.85 & 10.52 & -19.75 & 9.86 & -13.75 & 9.55 & -13.75 \\
\hline 9.52 & -5.95 & 9.86 & -11.65 & 9.76 & -7.05 & 9.65 & -9.45 & 9.44 & -12.05 \\
\hline 9.11 & -2.95 & 9.26 & -5.95 & 8.63 & 2.25 & 9.52 & -7.75 & 9.32 & -10.25 \\
\hline 8.60 & -0.55 & 8.68 & -2.15 & 8.12 & 5.15 & 9.35 & -5.55 & 9.20 & -8.95 \\
\hline 8.35 & 0.85 & 7.74 & 1.55 & 7.60 & 7.35 & 9.20 & -4.45 & 9.08 & -7.65 \\
\hline 8.08 & 1.85 & 6.74 & 4.75 & 7.05 & 9.05 & 8.94 & -2.25 & 8.87 & -6.35 \\
\hline 7.55 & 3.75 & 5.64 & 6.55 & 6.63 & 10.25 & 8.70 & -0.75 & 8.57 & -4.55 \\
\hline 7.48 & 3.75 & 4.60 & 6.95 & 6.10 & 11.45 & 8.22 & 2.15 & 8.20 & -2.65 \\
\hline 7.11 & 4.75 & 3.60 & 6.25 & 5.61 & 12.05 & 7.70 & 4.05 & 7.94 & -1.75 \\
\hline 6.60 & 6.15 & 2.10 & 2.95 & 5.11 & 12.85 & 7.19 & 6.05 & 7.60 & -0.15 \\
\hline 6.10 & 7.05 & 1.62 & 0.65 & 4.60 & 13.25 & 6.67 & 7.15 & 7.31 & 0.45 \\
\hline 5.57 & 7.75 & 1.10 & -2.25 & 4.11 & 13.45 & 5.72 & 8.45 & 6.82 & 1.95 \\
\hline 5.15 & 8.15 & 0.62 & -7.35 & 3.65 & 13.05 & 5.14 & 8.85 & 6.48 & 2.85 \\
\hline 4.63 & 8.25 & & & 3.13 & 12.45 & 4.88 & 8.95 & 6.01 & 3.25 \\
\hline 4.11 & 7.85 & & & 2.61 & 11.45 & 4.25 & 8.55 & 5.55 & 3.55 \\
\hline 3.59 & 7.35 & & & 2.12 & 9.85 & 4.00 & 8.45 & 5.30 & 3.95 \\
\hline 3.10 & 6.65 & & & 1.58 & 7.05 & 3.48 & 7.45 & 4.72 & 4.15 \\
\hline 2.60 & 5.25 & & & 1.08 & 3.25 & 2.89 & 6.15 & 4.16 & 4.15 \\
\hline 2.08 & 3.25 & & & & & 2.62 & 5.45 & 3.70 & 3.75 \\
\hline \multirow[t]{9}{*}{1.59} & 0.15 & & & & & 2.34 & 4.15 & 3.20 & 3.05 \\
\hline & & & & & & 2.08 & 3.15 & 2.76 & 2.05 \\
\hline & & & & & & 1.56 & -0.15 & 2.24 & 0.55 \\
\hline & & & & & & 1.12 & -3.75 & 1.69 & -1.75 \\
\hline & & & & & & 0.62 & -10.75 & 1.40 & -3.75 \\
\hline & & & & & & 0.36 & -17.05 & 1.21 & -5.05 \\
\hline & & & & & & & & 0.88 & -8.25 \\
\hline & & & & & & & & 0.54 & -13.15 \\
\hline & & & & & & & & 0.29 & -17.95 \\
\hline
\end{tabular}




\begin{tabular}{|c|c|c|c|c|c|c|c|c|c|}
\hline $\begin{array}{l}\text { SNG11 } \\
\text { /MPa }\end{array}$ & $\begin{array}{c}\text { SNG11 } \\
/{ }^{\circ} \mathrm{C}\end{array}$ & $\begin{array}{c}\text { SNG12 } \\
\text { /MPa }\end{array}$ & $\begin{array}{c}\text { SNG12 } \\
\quad /{ }^{\circ} \mathrm{C}\end{array}$ & $\begin{array}{c}\text { SNG13 } \\
/ \mathrm{MPa}\end{array}$ & $\begin{array}{c}\text { SNG13 } \\
\quad /{ }^{\circ} \mathrm{C}\end{array}$ & $\begin{array}{c}\text { SNG14 } \\
\text { /MPa }\end{array}$ & $\begin{array}{c}\text { SNG14 } \\
\quad /{ }^{\circ} \mathrm{C}\end{array}$ & $\begin{array}{l}\text { SNG15 } \\
\text { /MPa }\end{array}$ & $\begin{array}{c}\text { SNG15 } \\
/{ }^{\circ} \mathrm{C}\end{array}$ \\
\hline 9.72 & -11.15 & 0.23 & -43.45 & 0.25 & -67.35 & 0.47 & -36.35 & 0.19 & -25.45 \\
\hline 9.68 & -9.85 & 0.49 & -36.75 & 0.55 & -61.05 & 0.89 & -31.35 & 0.30 & -20.35 \\
\hline 9.54 & -7.65 & 0.99 & -31.35 & 1.00 & -53.65 & 1.51 & -28.15 & 0.52 & -12.45 \\
\hline 9.42 & -5.95 & 1.49 & -28.75 & 1.49 & -48.65 & 2.00 & -26.65 & 0.92 & -6.65 \\
\hline 9.32 & -4.45 & 1.97 & -27.85 & 1.94 & -45.75 & 2.50 & -26.75 & 1.44 & -1.85 \\
\hline 9.13 & -1.85 & 2.51 & -27.65 & 2.48 & -44.65 & 3.00 & -27.65 & 1.99 & 0.95 \\
\hline 8.74 & 0.65 & 2.93 & -27.95 & 2.98 & -44.05 & 3.56 & -28.45 & 2.50 & 2.65 \\
\hline 8.50 & 2.55 & 3.46 & -29.05 & 3.43 & -44.45 & 4.00 & -29.85 & 2.94 & 3.45 \\
\hline 8.26 & 4.45 & 3.93 & -30.65 & 4.06 & -44.65 & 4.50 & -31.65 & 3.50 & 3.95 \\
\hline 7.85 & 5.95 & 4.41 & -32.35 & 4.50 & -45.15 & 5.03 & -34.25 & 3.93 & 4.15 \\
\hline 7.42 & 7.85 & 4.90 & -34.75 & 4.97 & -45.15 & 5.47 & -37.35 & 4.45 & 3.85 \\
\hline 6.82 & 9.95 & 5.41 & -38.25 & 5.39 & -45.45 & 5.90 & -43.15 & 4.95 & 3.35 \\
\hline 6.40 & 11.05 & 5.91 & -43.75 & 5.88 & -46.55 & 6.41 & -51.95 & 5.48 & 2.35 \\
\hline 5.72 & 12.15 & 6.24 & -47.75 & 6.58 & -48.25 & 6.38 & -59.75 & 6.00 & 1.65 \\
\hline 5.34 & 12.65 & 6.39 & -53.35 & 6.97 & -52.05 & 5.97 & -70.15 & 6.49 & 0.25 \\
\hline 4.95 & 13.15 & 6.35 & -60.05 & 6.75 & -59.35 & 5.47 & -76.15 & 7.03 & -0.75 \\
\hline 4.74 & 13.15 & 6.23 & -63.15 & 6.27 & -61.55 & 5.04 & -77.65 & 7.59 & -2.25 \\
\hline 4.24 & 13.15 & 5.96 & -68.45 & & & & & 8.11 & -4.35 \\
\hline 3.73 & 12.95 & & & & & & & & \\
\hline 3.14 & 12.25 & & & & & & & & \\
\hline 2.92 & 11.95 & & & & & & & & \\
\hline 2.31 & 10.45 & & & & & & & & \\
\hline 2.00 & 9.35 & & & & & & & & \\
\hline 1.49 & 6.75 & & & & & & & & \\
\hline 1.11 & 3.75 & & & & & & & & \\
\hline 0.56 & -2.75 & & & & & & & & \\
\hline
\end{tabular}




\begin{tabular}{|c|c|c|c|c|c|c|c|c|c|}
\hline $\begin{array}{l}\text { SNG16 } \\
\text { /MPa }\end{array}$ & $\begin{array}{c}\text { SNG16 } \\
/{ }^{\circ} \mathrm{C} \\
\end{array}$ & $\begin{array}{c}\text { SNG17 } \\
\text { /MPa }\end{array}$ & $\begin{array}{c}\text { SNG17 } \\
/{ }^{\circ} \mathrm{C} \\
\end{array}$ & $\begin{array}{c}\text { SNG18 } \\
/ \mathrm{MPa}\end{array}$ & $\begin{array}{c}\text { SNG18 } \\
\quad /{ }^{\circ} \mathrm{C} \\
\end{array}$ & $\begin{array}{c}\text { SNG19 } \\
/ \mathrm{MPa}\end{array}$ & $\begin{array}{c}\text { SNG19 } \\
/{ }^{\circ} \mathrm{C} \\
\end{array}$ & $\begin{array}{c}\text { SNG20 } \\
\text { /MPa }\end{array}$ & $\begin{array}{c}\mathrm{SN} 20 \\
/{ }^{\circ} \mathrm{C}\end{array}$ \\
\hline 0.20 & -29.75 & 0.54 & -72.85 & 0.29 & -70.25 & 0.23 & -73.25 & 0.47 & -69.55 \\
\hline 0.34 & -23.35 & 1.02 & -65.85 & 0.51 & -62.55 & 0.45 & -65.75 & 0.95 & -63.25 \\
\hline 0.50 & -19.05 & 1.47 & -60.25 & 0.70 & -58.55 & 0.63 & -59.35 & 1.43 & -59.25 \\
\hline 0.70 & -14.85 & 1.96 & -55.05 & 1.00 & -54.65 & 1.03 & -53.05 & 1.97 & -56.75 \\
\hline 0.99 & -10.75 & 2.45 & -53.55 & 1.49 & -48.45 & 1.50 & -47.95 & 2.47 & -55.25 \\
\hline 1.47 & -6.45 & 2.97 & -51.45 & 1.99 & -44.25 & 2.00 & -43.05 & 3.02 & -54.25 \\
\hline 2.01 & -3.45 & 3.57 & -49.45 & 2.55 & -41.05 & 2.46 & -40.05 & 3.47 & -54.35 \\
\hline 2.46 & -1.75 & 4.04 & -48.45 & 3.01 & -39.15 & 2.96 & -37.65 & 3.95 & -55.45 \\
\hline 3.06 & -0.45 & 4.44 & -47.65 & 3.51 & -37.65 & 3.55 & -35.45 & 4.49 & -55.35 \\
\hline 3.51 & 0.05 & 4.95 & -47.55 & 4.02 & -36.75 & 4.03 & -33.85 & 5.09 & -56.35 \\
\hline 4.00 & 0.15 & 5.58 & -46.75 & 4.50 & -35.75 & 4.54 & -32.95 & 5.47 & -58.25 \\
\hline 4.50 & 0.35 & 6.11 & -46.25 & 4.90 & -35.45 & 5.00 & -32.35 & 5.84 & -61.55 \\
\hline 5.00 & 0.05 & 6.47 & -46.55 & 5.50 & -35.75 & 5.45 & -32.35 & 5.83 & -67.15 \\
\hline 5.50 & -0.65 & 6.67 & -50.75 & 6.05 & -36.05 & 5.95 & -33.55 & 5.61 & -69.75 \\
\hline 6.01 & -1.35 & 6.37 & -55.65 & 6.52 & -37.85 & 6.34 & -33.75 & & \\
\hline 6.62 & -3.75 & & & 7.04 & -40.75 & 6.77 & -33.75 & & \\
\hline 7.14 & -5.55 & & & 7.12 & -45.25 & 7.05 & -34.75 & & \\
\hline 7.53 & -8.55 & & & 6.94 & -50.95 & 7.45 & -36.45 & & \\
\hline 8.02 & -11.05 & & & 6.40 & -55.55 & 7.74 & -39.35 & & \\
\hline 8.48 & -15.25 & & & & & 7.77 & -43.95 & & \\
\hline 8.85 & -21.05 & & & & & 7.36 & -52.75 & & \\
\hline 9.14 & -27.05 & & & & & 6.96 & -57.35 & & \\
\hline 9.23 & -31.25 & & & & & & & & \\
\hline 9.05 & -41.75 & & & & & & & & \\
\hline 8.87 & -45.05 & & & & & & & & \\
\hline 8.28 & -49.85 & & & & & & & & \\
\hline 7.42 & -57.45 & & & & & & & & \\
\hline
\end{tabular}




\begin{tabular}{|c|c|c|c|c|c|c|c|c|c|}
\hline $\begin{array}{c}\text { SNG21 } \\
\text { /MPa }\end{array}$ & $\begin{array}{c}\text { SNG21 } \\
/{ }^{\circ} \mathrm{C}\end{array}$ & $\begin{array}{c}\text { SNG22 } \\
/ \mathrm{MPa}\end{array}$ & $\begin{array}{c}\text { SNG22 } \\
/{ }^{\circ} \mathrm{C}\end{array}$ & $\begin{array}{c}\text { SNG23 } \\
\text { /MPa }\end{array}$ & $\begin{array}{c}\text { SNG23 } \\
/{ }^{\circ} \mathrm{C}\end{array}$ & $\begin{array}{c}\text { SNG24 } \\
\text { /MPa }\end{array}$ & $\begin{array}{c}\text { SNG24 } \\
/{ }^{\circ} \mathrm{C}\end{array}$ & $\begin{array}{c}\text { SNG25 } \\
\text { /MPa }\end{array}$ & $\begin{array}{c}\text { SNG25 } \\
/{ }^{\circ} \mathrm{C}\end{array}$ \\
\hline 0.12 & -59.55 & 0.12 & -55.25 & 1.27 & -19.45 & 8.80 & -37.50 & 9.40 & -25.43 \\
\hline 0.12 & -56.95 & 0.14 & -53.55 & 1.29 & -19.15 & 8.50 & -31.57 & 9.00 & -19.73 \\
\hline 0.12 & -56.75 & 0.21 & -50.55 & 1.31 & -18.85 & 8.00 & -25.33 & 8.00 & -11.88 \\
\hline 0.12 & -56.15 & 0.31 & -45.05 & 1.33 & -18.65 & 7.50 & -21.60 & 7.00 & -7.27 \\
\hline 0.13 & -55.65 & 0.41 & -41.05 & 1.35 & -18.45 & 7.00 & -17.30 & 6.00 & -4.05 \\
\hline 0.13 & -54.55 & 0.53 & -37.25 & 1.37 & -18.15 & 6.00 & -13.17 & 5.00 & -2.30 \\
\hline 0.14 & -53.65 & 0.64 & -34.45 & 1.38 & -17.95 & 5.00 & -9.37 & 4.00 & -1.53 \\
\hline 0.16 & -52.05 & 0.73 & -32.45 & 1.40 & -17.75 & 4.00 & -7.13 & 3.00 & -2.13 \\
\hline 0.17 & -51.15 & 0.86 & -30.65 & 1.42 & -17.55 & 3.00 & -6.33 & & \\
\hline 0.18 & -50.45 & 0.98 & -28.75 & 1.44 & -17.25 & 2.00 & -7.67 & & \\
\hline 0.21 & -48.65 & 1.18 & -26.05 & 1.46 & -17.05 & 1.50 & -9.67 & & \\
\hline 0.25 & -46.55 & 1.21 & -25.65 & 1.48 & -16.85 & 1.00 & -13.37 & & \\
\hline 0.29 & -44.65 & 1.36 & -23.95 & 1.50 & -16.65 & & & & \\
\hline 0.31 & -43.85 & 1.48 & -22.75 & 1.52 & -16.45 & & & & \\
\hline 0.38 & -41.65 & 1.75 & -20.85 & 1.53 & -16.25 & & & & \\
\hline 0.41 & -40.55 & 1.91 & -19.65 & 1.55 & -16.05 & & & & \\
\hline 0.44 & -39.75 & 2.19 & -18.45 & 1.57 & -15.85 & & & & \\
\hline 0.50 & -38.05 & 2.52 & -16.75 & 1.59 & -15.55 & & & & \\
\hline 0.52 & -37.55 & 2.89 & -15.15 & 1.61 & -15.35 & & & & \\
\hline 0.58 & -36.45 & 3.05 & -14.65 & 1.70 & -14.55 & & & & \\
\hline 0.63 & -35.35 & 3.40 & -13.65 & 1.74 & -14.15 & & & & \\
\hline 0.67 & -34.75 & 3.75 & -13.05 & 1.82 & -13.35 & & & & \\
\hline 0.72 & -33.75 & 4.18 & -12.35 & 1.93 & -12.45 & & & & \\
\hline 0.79 & -32.75 & 4.52 & -12.05 & 2.03 & -11.65 & & & & \\
\hline 0.85 & -31.85 & 5.10 & -11.75 & 2.13 & -10.85 & & & & \\
\hline 0.92 & -31.05 & 5.73 & -12.35 & 2.23 & -10.05 & & & & \\
\hline
\end{tabular}




\begin{tabular}{|c|c|c|c|c|c|c|c|c|c|}
\hline SNG21 & SNG21 & SNG22 & SNG22 & SNG23 & SNG23 & SNG24 & SNG24 & SNG25 & SNG25 \\
\hline /MPa & $/{ }^{\circ} \mathrm{C}$ & /MPa & $/{ }^{\circ} \mathrm{C}$ & $/ \mathrm{MPa}$ & $/{ }^{\circ} \mathrm{C}$ & /MPa & $/{ }^{\circ} \mathrm{C}$ & /MPa & $/{ }^{\circ} \mathrm{C}$ \\
\hline 1.01 & -29.95 & 6.24 & -13.25 & 2.34 & -9.35 & & & & \\
\hline 1.10 & -29.05 & 6.51 & -14.05 & 2.45 & -8.65 & & & & \\
\hline 1.20 & -28.05 & 6.76 & -15.05 & 2.55 & -7.95 & & & & \\
\hline 1.29 & -27.35 & 6.98 & -16.65 & 2.66 & -7.35 & & & & \\
\hline 1.42 & -26.45 & 7.19 & -17.25 & 2.76 & -6.75 & & & & \\
\hline 1.52 & -25.75 & 7.54 & -18.95 & 2.87 & -6.25 & & & & \\
\hline 1.80 & -24.25 & 7.59 & -19.65 & 2.98 & -5.75 & & & & \\
\hline 1.94 & -23.65 & 7.77 & -20.75 & 3.09 & -5.15 & & & & \\
\hline 2.02 & -23.35 & 7.78 & -21.75 & 3.19 & -4.75 & & & & \\
\hline 2.09 & -23.05 & 7.92 & -39.15 & 3.30 & -4.35 & & & & \\
\hline 2.22 & -22.65 & 7.93 & -38.35 & 3.40 & -3.95 & & & & \\
\hline 2.30 & -22.45 & 7.94 & -22.85 & 3.51 & -3.55 & & & & \\
\hline 2.42 & -22.05 & 8.00 & -36.95 & 3.61 & -3.15 & & & & \\
\hline 2.53 & -21.85 & 8.07 & -25.85 & 3.71 & -2.85 & & & & \\
\hline 2.64 & -21.65 & 8.10 & -34.95 & 3.82 & -2.45 & & & & \\
\hline 2.76 & -21.45 & 8.13 & -33.55 & 3.93 & -2.25 & & & & \\
\hline 2.88 & -21.25 & 8.13 & -31.45 & 4.01 & -2.05 & & & & \\
\hline 3.04 & -21.15 & 8.13 & -27.85 & 4.10 & -1.85 & & & & \\
\hline 3.14 & -21.05 & 8.17 & -30.15 & 4.19 & -1.65 & & & & \\
\hline 3.25 & -20.95 & 8.18 & -30.85 & 4.28 & -1.35 & & & & \\
\hline 3.37 & -20.95 & 8.18 & -28.65 & 4.37 & -1.25 & & & & \\
\hline 3.40 & -20.95 & & & 4.47 & -1.05 & & & & \\
\hline 3.53 & -20.95 & & & 4.57 & -0.85 & & & & \\
\hline 3.68 & -20.95 & & & 4.67 & -0.65 & & & & \\
\hline 3.83 & -20.95 & & & 4.77 & -0.55 & & & & \\
\hline 3.96 & -21.15 & & & 4.87 & -0.45 & & & & \\
\hline
\end{tabular}




\begin{tabular}{|c|c|c|c|c|c|c|c|c|c|}
\hline SNG21 & SNG21 & SNG22 & SNG22 & SNG23 & SNG23 & SNG24 & SNG24 & SNG25 & SNG25 \\
\hline /MPa & $/{ }^{\circ} \mathrm{C}$ & $/ \mathrm{MPa}$ & $/{ }^{\circ} \mathrm{C}$ & /MPa & $/{ }^{\circ} \mathrm{C}$ & /MPa & $/{ }^{\circ} \mathrm{C}$ & /MPa & $1{ }^{\circ} \mathrm{C}$ \\
\hline 4.08 & -21.25 & & & 4.97 & -0.35 & & & & \\
\hline 4.30 & -21.55 & & & 5.05 & -0.25 & & & & \\
\hline 4.50 & -21.85 & & & 5.14 & -0.15 & & & & \\
\hline 4.63 & -22.15 & & & 5.22 & -0.05 & & & & \\
\hline 4.79 & -22.45 & & & 5.31 & -0.05 & & & & \\
\hline 5.00 & -22.95 & & & 5.40 & 0.05 & & & & \\
\hline 5.20 & -23.65 & & & 5.50 & 0.05 & & & & \\
\hline 5.42 & -24.35 & & & 5.59 & 0.15 & & & & \\
\hline 5.61 & -25.05 & & & 5.69 & 0.15 & & & & \\
\hline 5.83 & -25.55 & & & 5.78 & 0.15 & & & & \\
\hline \multirow[t]{16}{*}{6.02} & -26.75 & & & 5.87 & 0.15 & & & & \\
\hline & & & & 5.96 & 0.15 & & & & \\
\hline & & & & 6.06 & 0.15 & & & & \\
\hline & & & & 6.15 & 0.15 & & & & \\
\hline & & & & 6.24 & 0.15 & & & & \\
\hline & & & & 6.34 & 0.05 & & & & \\
\hline & & & & 6.44 & 0.05 & & & & \\
\hline & & & & 6.53 & -0.05 & & & & \\
\hline & & & & 6.62 & -0.15 & & & & \\
\hline & & & & 6.71 & -0.25 & & & & \\
\hline & & & & 6.80 & -0.25 & & & & \\
\hline & & & & 6.90 & -0.45 & & & & \\
\hline & & & & 6.99 & -0.45 & & & & \\
\hline & & & & 7.08 & -0.55 & & & & \\
\hline & & & & 7.17 & -0.75 & & & & \\
\hline & & & & 7.27 & -0.95 & & & & \\
\hline
\end{tabular}




\begin{tabular}{|c|c|c|c|c|c|c|c|c|c|}
\hline $\begin{array}{l}\text { SNG21 } \\
\text { /MPa }\end{array}$ & $\begin{array}{c}\text { SNG21 } \\
/{ }^{\circ} \mathrm{C}\end{array}$ & $\begin{array}{c}\text { SNG22 } \\
/ \mathrm{MPa}\end{array}$ & $\begin{array}{c}\text { SNG22 } \\
/{ }^{\circ} \mathrm{C}\end{array}$ & $\begin{array}{c}\text { SNG23 } \\
/ \mathrm{MPa}\end{array}$ & $\begin{array}{c}\text { SNG23 } \\
/{ }^{\circ} \mathrm{C}\end{array}$ & $\begin{array}{c}\text { SNG24 } \\
/ \mathrm{MPa}\end{array}$ & $\begin{array}{c}\text { SNG24 } \\
/{ }^{\circ} \mathrm{C}\end{array}$ & $\begin{array}{c}\text { SNG25 } \\
/ \mathrm{MPa}\end{array}$ & $\begin{array}{c}\text { SNG25 } \\
/{ }^{\circ} \mathrm{C}\end{array}$ \\
\hline & & & & 7.36 & -1.05 & & & & \\
\hline & & & & 7.46 & -1.25 & & & & \\
\hline & & & & 7.55 & -1.35 & & & & \\
\hline & & & & 7.64 & -1.55 & & & & \\
\hline & & & & 7.73 & -1.75 & & & & \\
\hline & & & & 7.82 & -1.95 & & & & \\
\hline & & & & 7.92 & -2.25 & & & & \\
\hline & & & & 8.01 & -2.45 & & & & \\
\hline & & & & 8.09 & -2.65 & & & & \\
\hline & & & & 8.19 & -2.95 & & & & \\
\hline & & & & 8.28 & -3.25 & & & & \\
\hline & & & & 8.37 & -3.55 & & & & \\
\hline & & & & 8.46 & -3.85 & & & & \\
\hline & & & & 8.55 & -4.25 & & & & \\
\hline & & & & 8.64 & -4.65 & & & & \\
\hline & & & & 8.73 & -5.05 & & & & \\
\hline & & & & 8.82 & -5.45 & & & & \\
\hline & & & & 8.91 & -5.85 & & & & \\
\hline & & & & 9.00 & -6.25 & & & & \\
\hline & & & & 9.08 & -6.55 & & & & \\
\hline & & & & 9.17 & -7.05 & & & & \\
\hline & & & & 9.25 & -7.35 & & & & \\
\hline
\end{tabular}


Table S4. Measured hydrocarbon dew point of 13 RGs from other literatures

\begin{tabular}{|c|c|c|c|c|c|c|c|c|c|}
\hline RG1 & RG1 & RG2 & RG2 & RG3 & RG3 & RG4 & RG4 & RG5 & RG5 \\
\hline$/ \mathrm{MPa}$ & $/{ }^{\circ} \mathrm{C}$ & $/ \mathrm{MPa}$ & $/{ }^{\circ} \mathrm{C}$ & $/ \mathrm{MPa}$ & $/{ }^{\circ} \mathrm{C}$ & $/ \mathrm{MPa}$ & $/{ }^{\circ} \mathrm{C}$ & $/ \mathrm{MPa}$ & $/{ }^{\circ} \mathrm{C}$ \\
\hline 10.83 & -9.45 & 10.76 & -13.95 & 10.90 & -10.55 & 10.96 & -11.35 & 10.50 & -8.95 \\
\hline 10.69 & -5.55 & 10.68 & -9.55 & 10.80 & -7.05 & 10.92 & -10.15 & 10.49 & -8.65 \\
\hline 10.53 & -2.25 & 10.55 & -6.45 & 10.75 & -3.45 & 10.75 & -4.95 & 10.48 & -9.45 \\
\hline 10.31 & 1.65 & 10.43 & -3.25 & 10.70 & -1.45 & 10.50 & -0.45 & 10.47 & -8.15 \\
\hline 10.05 & 4.95 & 10.31 & -0.55 & 10.55 & 1.15 & 10.10 & 3.45 & 10.45 & -7.25 \\
\hline 9.55 & 7.75 & 10.13 & 0.65 & 10.40 & 2.45 & 9.06 & 10.85 & 10.37 & -4.75 \\
\hline 9.10 & 10.15 & 9.64 & 5.45 & 10.10 & 5.05 & 8.10 & 14.15 & 10.35 & -4.45 \\
\hline 8.07 & 13.85 & 9.05 & 8.85 & 9.90 & 6.05 & 7.02 & 17.25 & 10.34 & -3.95 \\
\hline 7.05 & 15.65 & 8.09 & 13.05 & 9.60 & 7.55 & 6.15 & 19.05 & 10.33 & -3.75 \\
\hline 6.08 & 17.45 & 7.08 & 15.15 & 9.05 & 11.95 & 5.00 & 20.05 & 10.18 & -0.65 \\
\hline 5.02 & 18.45 & 6.07 & 17.25 & 8.55 & 14.95 & 4.10 & 19.75 & 10.17 & -0.75 \\
\hline 3.98 & 18.45 & 5.03 & 18.05 & 8.08 & 16.95 & 3.07 & 18.55 & 10.16 & 0.15 \\
\hline \multirow[t]{13}{*}{2.99} & 17.05 & 4.47 & 18.05 & 7.55 & 18.75 & & & 10.01 & 1.95 \\
\hline & & 4.00 & 17.75 & 6.85 & 20.75 & & & 10.00 & 2.15 \\
\hline & & 3.11 & 16.35 & 6.05 & 22.65 & & & 9.79 & 4.85 \\
\hline & & & & 5.55 & 23.65 & & & 9.15 & 10.05 \\
\hline & & & & 5.50 & 23.75 & & & 8.19 & 12.95 \\
\hline & & & & 5.00 & 23.95 & & & 7.11 & 16.15 \\
\hline & & & & & & & & 6.12 & 17.65 \\
\hline & & & & & & & & 6.11 & 17.75 \\
\hline & & & & & & & & 5.15 & 18.25 \\
\hline & & & & & & & & 4.14 & 18.05 \\
\hline & & & & & & & & 3.13 & 16.65 \\
\hline & & & & & & & & 2.17 & 13.35 \\
\hline & & & & & & & & 1.19 & 7.45 \\
\hline
\end{tabular}




\begin{tabular}{|c|c|c|c|c|c|c|c|c|c|}
\hline $\begin{array}{l}\text { RG6 } \\
\text { /MPa }\end{array}$ & $\begin{array}{c}\text { RG6 } \\
/{ }^{\circ} \mathrm{C}\end{array}$ & $\begin{array}{l}\text { RG7 } \\
\text { /MPa }\end{array}$ & $\begin{array}{c}\text { RG7 } \\
/{ }^{\circ} \mathrm{C}\end{array}$ & $\begin{array}{l}\text { RG8 } \\
\text { /MPa }\end{array}$ & $\begin{array}{l}\text { RG8 } \\
/{ }^{\circ} \mathrm{C}\end{array}$ & $\begin{array}{l}\text { RG9 } \\
\text { /MPa }\end{array}$ & $\begin{array}{l}\text { RG9 } \\
/{ }^{\circ} \mathrm{C}\end{array}$ & $\begin{array}{l}\text { RG10 } \\
\text { /MPa }\end{array}$ & $\begin{array}{c}\text { RG10 } \\
/{ }^{\circ} \mathrm{C}\end{array}$ \\
\hline 10.59 & -8.35 & 0.22 & -29.95 & 0.11 & -36.25 & 0.21 & -33.15 & 0.15 & -34.45 \\
\hline 10.58 & -7.45 & 0.26 & -21.65 & 0.24 & -25.35 & 0.36 & -26.55 & 0.26 & -28.85 \\
\hline 10.57 & -7.05 & 0.39 & -16.05 & 0.34 & -21.25 & 0.56 & -22.05 & 0.37 & -24.85 \\
\hline 10.55 & -6.35 & 0.47 & -13.55 & 0.54 & -16.05 & 0.67 & -20.35 & 0.52 & -21.15 \\
\hline 10.52 & -4.05 & 0.59 & -10.85 & 0.72 & -12.75 & 0.75 & -19.15 & 0.65 & -18.95 \\
\hline 10.51 & -3.25 & 0.73 & -8.45 & 0.94 & -9.45 & 0.93 & -17.05 & 0.83 & -16.45 \\
\hline 10.46 & -3.75 & 0.80 & -7.25 & 1.04 & -8.15 & 1.06 & -15.75 & 1.01 & -14.65 \\
\hline 10.43 & -2.85 & 0.97 & -5.15 & 1.25 & -6.25 & 1.30 & -14.25 & 1.22 & -12.85 \\
\hline 10.31 & -1.15 & 1.06 & -4.35 & 1.42 & -4.95 & 1.52 & -13.15 & 1.41 & -11.65 \\
\hline 10.30 & -0.85 & 1.12 & -3.55 & 1.61 & -3.75 & 1.74 & -12.15 & 1.66 & -10.35 \\
\hline 10.16 & 1.15 & 1.29 & -2.15 & 1.74 & -2.85 & 1.92 & -11.65 & 1.80 & -9.75 \\
\hline 10.15 & 1.25 & 1.41 & -1.35 & 1.95 & -1.85 & 2.16 & -11.15 & 2.09 & -8.85 \\
\hline 9.84 & 5.25 & 1.56 & -0.35 & 2.17 & -1.05 & 2.35 & -10.85 & 2.33 & -8.25 \\
\hline 9.61 & 6.35 & 1.65 & 0.15 & 2.30 & -0.55 & 2.54 & -10.55 & 2.58 & -7.75 \\
\hline 9.19 & 9.25 & 1.77 & 0.85 & 2.48 & 0.05 & 2.70 & -10.35 & 2.77 & -7.45 \\
\hline 9.18 & 9.25 & 1.89 & 1.45 & 2.72 & 0.55 & 2.91 & -10.15 & 2.99 & -7.25 \\
\hline 8.23 & 13.35 & 2.04 & 2.05 & 2.92 & 0.85 & 3.13 & -10.15 & 3.25 & -7.15 \\
\hline 8.22 & 13.25 & 2.13 & 2.35 & 3.09 & 1.25 & 3.35 & -10.25 & 3.53 & -7.15 \\
\hline 7.26 & 16.15 & 2.23 & 2.65 & 3.23 & 1.35 & 3.54 & -10.25 & 3.80 & -7.55 \\
\hline 7.25 & 16.25 & 2.34 & 3.05 & 3.37 & 1.45 & 3.71 & -10.35 & 4.08 & -8.25 \\
\hline 6.19 & 18.35 & 2.48 & 3.35 & 3.55 & 1.55 & 3.97 & -10.65 & 4.29 & -8.75 \\
\hline 6.18 & 18.45 & 2.60 & 3.65 & 3.76 & 1.55 & 4.16 & -11.05 & 4.50 & -9.15 \\
\hline 5.19 & 19.25 & 2.72 & 3.85 & 3.98 & 1.45 & 4.35 & -11.45 & 4.79 & -9.75 \\
\hline 4.12 & 18.95 & 2.85 & 4.05 & 4.20 & 1.25 & 4.51 & -11.95 & 5.01 & -10.25 \\
\hline 3.14 & 17.35 & 2.97 & 4.25 & 4.40 & 1.05 & 4.75 & -12.45 & 5.26 & -10.55 \\
\hline
\end{tabular}




\begin{tabular}{|c|c|c|c|c|c|c|c|c|c|}
\hline RG6 & RG6 & RG7 & RG7 & RG8 & RG8 & RG9 & RG9 & RG10 & RG10 \\
\hline /MPa & $/{ }^{\circ} \mathrm{C}$ & /MPa & $/{ }^{\circ} \mathrm{C}$ & $/ \mathrm{MPa}$ & $/{ }^{\circ} \mathrm{C}$ & $/ \mathrm{MPa}$ & $/{ }^{\circ} \mathrm{C}$ & $/ \mathrm{MPa}$ & $/{ }^{\circ} \mathrm{C}$ \\
\hline \multirow[t]{16}{*}{1.61} & 11.35 & 3.14 & 4.45 & 4.58 & 0.75 & 5.03 & -13.55 & 5.49 & -10.05 \\
\hline & & 3.33 & 4.55 & 4.88 & 0.25 & 5.18 & -14.25 & 5.71 & -10.55 \\
\hline & & 3.52 & 4.65 & 5.02 & -0.15 & 5.42 & -14.85 & & \\
\hline & & 3.68 & 4.75 & 5.22 & -0.55 & 5.60 & -15.15 & & \\
\hline & & 3.87 & 4.65 & 5.46 & -1.15 & & & & \\
\hline & & 4.11 & 4.55 & 5.68 & -1.85 & & & & \\
\hline & & 4.24 & 4.35 & 5.88 & -2.45 & & & & \\
\hline & & 4.40 & 4.25 & 6.02 & -2.95 & & & & \\
\hline & & 4.54 & 4.05 & 6.25 & -3.85 & & & & \\
\hline & & 4.68 & 3.85 & 6.48 & -4.75 & & & & \\
\hline & & 4.85 & 3.55 & 6.79 & -6.15 & & & & \\
\hline & & 5.09 & 3.15 & 6.96 & -7.15 & & & & \\
\hline & & 5.39 & 2.55 & 7.23 & -8.55 & & & & \\
\hline & & 5.69 & 1.85 & 7.43 & -9.95 & & & & \\
\hline & & 5.95 & 1.15 & 7.84 & -12.35 & & & & \\
\hline & & 6.16 & 0.65 & & & & & & \\
\hline
\end{tabular}

\begin{tabular}{cccccc}
\hline RG11 & RG11 & RG12 & RG12 & RG13 & RG13 \\
MPa & ${ }^{\circ} \mathrm{C}$ & MPa & ${ }^{\circ} \mathrm{C}$ & MPa & ${ }^{\circ} \mathrm{C}$ \\
\hline 0.11 & -36.65 & 0.11 & -37.95 & 10.50 & -1.37 \\
0.20 & -29.35 & 0.27 & -26.95 & 10.00 & 6.40 \\
0.36 & -22.05 & 0.42 & -22.25 & 9.00 & 14.40 \\
0.47 & -18.85 & 0.60 & -18.35 & 8.00 & 18.50 \\
0.61 & -16.15 & 0.74 & -16.05 & 7.00 & 21.13 \\
0.72 & -14.25 & 0.91 & -13.75 & 6.00 & 22.83
\end{tabular}




\begin{tabular}{cccccc}
\hline $\begin{array}{c}\text { RG11 } \\
\text { MPa }\end{array}$ & $\begin{array}{c}\text { RG11 } \\
{ }^{\circ} \mathrm{C}\end{array}$ & $\begin{array}{c}\text { RG12 } \\
\text { MPa }\end{array}$ & $\begin{array}{c}\text { RG12 } \\
{ }^{\circ} \mathrm{C}\end{array}$ & $\begin{array}{c}\text { RG13 } \\
\text { MPa }\end{array}$ & $\begin{array}{c}\text { RG13 } \\
{ }^{\circ} \mathrm{C}\end{array}$ \\
\hline 0.87 & -12.05 & 1.12 & -11.85 & 5.00 & 23.33 \\
1.03 & -10.45 & 1.28 & -10.55 & & \\
1.20 & -8.85 & 1.44 & -9.45 & & \\
1.38 & -7.55 & 1.66 & -8.15 & & \\
1.47 & -6.65 & 1.85 & -7.25 & & \\
1.80 & -5.35 & 2.03 & -6.55 & \\
1.94 & -4.65 & 2.21 & -5.85 & \\
2.13 & -3.95 & 2.41 & -5.35 & \\
2.35 & -3.35 & 2.64 & -4.75 & \\
2.51 & -2.95 & 2.85 & -4.45 & \\
2.71 & -2.55 & 3.05 & -4.25 & \\
2.94 & -2.25 & 3.26 & -4.05 & \\
3.14 & -2.15 & 3.46 & -3.95 & \\
3.35 & -2.05 & 3.70 & -3.95 & \\
3.51 & -2.05 & 3.95 & -4.05 & \\
3.74 & -2.15 & 4.13 & -4.25 & \\
4.01 & -2.55 & 4.31 & -4.45 & \\
4.29 & -3.05 & 4.53 & -4.75 & \\
4.53 & -3.45 & 4.73 & -5.05 & \\
4.77 & -3.95 & 4.96 & -5.35 & \\
4.96 & -4.35 & 5.17 & -5.75 & \\
5.23 & -5.05 & 5.36 & -6.25 & \\
5.40 & -5.45 & 5.54 & -6.55 & \\
5.64 & -6.35 & 5.82 & -6.65 & \\
\hline
\end{tabular}


Table S5. The binary interaction parameters for SRK, SRK-Twu and PSRK

\begin{tabular}{|c|c|c|c|c|c|c|c|c|c|c|c|}
\hline & $\mathrm{He}$ & $\mathrm{H}_{2}$ & $\mathrm{~N}_{2}$ & $\mathrm{CO}_{2}$ & $\mathrm{CH}_{4}$ & $\mathrm{C}_{2} \mathrm{H}_{6}$ & $\mathrm{C}_{3} \mathrm{H}_{8}$ & $\mathrm{i}-\mathrm{C}_{4}$ & $\mathrm{n}-\mathrm{C}_{4}$ & neoC $_{5}$ & $\mathrm{i}-\mathrm{C}_{5}$ \\
\hline $\mathrm{He}$ & $<$ empty $>$ & 0.399300 & 0.094400 & 0.916100 & 0.750000 & 1.406900 & 1.249300 & 0.000000 & 0.000000 & 0.000000 & 0.000000 \\
\hline $\mathrm{H}_{2}$ & 0.399300 & <empty $>$ & -0.001000 & 0.116400 & 0.000100 & 0.000100 & 0.000100 & 0.000200 & 0.000200 & 0.000000 & 0.000200 \\
\hline $\mathrm{N}_{2}$ & 0.094400 & -0.001000 & <empty> & -0.017100 & 0.031199 & 0.031899 & 0.088600 & 0.131500 & 0.059700 & 0.100000 & 0.093000 \\
\hline $\mathrm{CH}_{4}$ & 750000 & 0.000100 & 0.031199 & 0.095600 & $<$ empty> & 0.002241 & 0.006829 & 0.013113 & 0.012305 & 0.017131 & 0.017628 \\
\hline $\mathrm{C}_{2} \mathrm{H}_{6}$ & 406900 & 0.000100 & 0.031899 & 0.140100 & 0.002241 & $<$ empty $>$ & 0.001258 & 0.004573 & 0.004096 & 0.007088 & 0.007413 \\
\hline$n-C_{4}$ & 0.000000 & 0.000200 & 0.059700 & 0.141200 & 0.012305 & 0.004096 & 0.000819 & 0.000013 & <empty> & 0.000413 & 0.000495 \\
\hline neoC $_{5}$ & 0.000000 & 0.000000 & 0.100000 & 0.108900 & 0.017131 & 0.007088 & 0.002392 & 0.000278 & 0.000413 & <empty $>$ & 0.000004 \\
\hline $\mathrm{i}-\mathrm{C}_{5}$ & .000000 & 0.000200 & 0.093000 & 0.129700 & 0.017628 & 0.007413 & 0.002583 & 0.000346 & 0.000495 & 0.000004 & <empty> \\
\hline$n-C_{5}$ & 0.000000 & 0.000200 & 0.093598 & 0.134700 & 0.017925 & 0.007609 & 0.002701 & 0.000390 & 0.000547 & 0.000009 & 0.000001 \\
\hline сус- $-C_{5}$ & 000000 & 0.000000 & 0.100000 & 0.108900 & 0.012809 & 0.004393 & 0.000955 & 0.000002 & 0.000005 & 0.000325 & 0.000399 \\
\hline$n-C_{6}$ & 0.000000 & 0.000200 & 0.165000 & 0.142000 & 0.023474 & 0.011414 & 0.005142 & 0.001565 & 0.001866 & 0.000524 & 0.000440 \\
\hline MCyc- $_{5}$ & 0.000000 & 0.000000 & 0.100000 & 0.108900 & 0.018718 & 0.008136 & 0.003020 & 0.000517 & 0.000696 & 0.000037 & 0.000017 \\
\hline benzene & 0.000000 & 0.000000 & 0.100000 & 0.080499 & 0.012809 & 0.028000 & 0.019997 & 0.000002 & 0.000005 & 0.000325 & 0.000399 \\
\hline cyclohexane & 0.000000 & 0.260100 & 0.100000 & 0.107100 & 0.031098 & 0.018300 & 0.010200 & 0.000346 & 0.000495 & 0.000004 & 0.000000 \\
\hline$n-C_{7}$ & 0.000000 & 0.000000 & 0.079989 & 0.109200 & 0.028864 & 0.015324 & 0.007887 & 0.003221 & 0.003646 & 0.001610 & 0.001459 \\
\hline $\mathrm{MCyc} \mathrm{C}_{6}$ & 0.000000 & 0.000000 & & 0.109300 & 0.023474 & 0.008000 & 0.005142 & 0.001565 & 0.001866 & 0.000524 & 0.000440 \\
\hline toluene & 0.000000 & 0.000000 & & 0.098200 & 0.054698 & 0.029998 & 0.014990 & 0.009999 & 0.009999 & 0.000024 & 0.004999 \\
\hline $\mathrm{Cyc}_{-} \mathrm{C}_{7}$ & 0.000000 & 0.000000 & 0.100000 & 0.108900 & 0.022036 & 0.010403 & 0.004466 & 0.001202 & 0.001467 & 0.000324 & 0.000258 \\
\hline$n-C_{8}$ & 0.000000 & 0.000000 & 0.079989 & 0.135000 & 0.034159 & 0.019319 & 0.010850 & 0.005214 & 0.005750 & 0.003093 & 0.002883 \\
\hline Сyc- $\mathrm{C}_{8}$ & 0.000000 & 0.000000 & 0.100000 & 0.108900 & 0.027405 & 0.014248 & 0.007114 & 0.002732 & 0.003124 & 0.001269 & 0.001135 \\
\hline $\mathrm{n}-\mathrm{C}_{9}$ & 0.000000 & 0.000000 & 0.079989 & 0.135000 & 0.038926 & 0.023017 & 0.013697 & 0.007255 & 0.007883 & 0.004708 & 0.004449 \\
\hline
\end{tabular}




\begin{tabular}{|c|c|c|c|c|c|c|c|c|c|c|c|}
\hline & $n-C_{5}$ & сус- $\mathrm{C}_{5}$ & $22 \mathrm{M}-\mathrm{C}_{4}$ & $23 \mathrm{M}-\mathrm{C}_{4}$ & $2 \mathrm{M}-\mathrm{C}_{5}$ & $3 \mathrm{M}-\mathrm{C}_{5}$ & $n-C_{6}$ & M-Сус- ${ }_{5}$ & benzene & cyclohexane & $\mathrm{n}-\mathrm{C}_{7}$ \\
\hline $\mathrm{He}$ & 0.000000 & 0.000000 & 0.000000 & 0.000000 & 0.000000 & 0.000000 & 0.000000 & 0.000000 & 0.000000 & 0.000000 & 0.000000 \\
\hline $\mathrm{H}_{2}$ & 000200 & 0.000000 & 0.000000 & 0.000000 & 0.000000 & 0.000000 & 0.000200 & 0.000000 & 0.000000 & 0.260100 & 0.000000 \\
\hline $\mathrm{N}_{2}$ & 093598 & 0.100000 & 0.100000 & 0.100000 & 0.100000 & 0.100000 & 0.165000 & 0.100000 & 0.100000 & 0.100000 & 0.079989 \\
\hline $\mathrm{CO}_{2}$ & 0.134700 & 0.108900 & 0.108900 & 0.108900 & 0.108900 & 0.108900 & 0.142000 & 0.108900 & 0.080499 & 0.107100 & 0.109200 \\
\hline $\mathrm{CH}_{4}$ & 0.017925 & 0.012809 & 0.022613 & 0.022518 & 0.023318 & 0.023318 & 0.023474 & 0.018718 & 0.012809 & 0.031098 & 0.028864 \\
\hline $\mathrm{C}_{2} \mathrm{H}_{6}$ & 007609 & 0.004393 & 0.010807 & 0.010740 & 0.011303 & 0.011303 & 0.011414 & 0.008136 & 0.028000 & 0.018300 & 0.015324 \\
\hline $\mathrm{n}-\mathrm{C}_{4}$ & 000547 & 0.000005 & 0.001623 & 0.001597 & 0.001821 & 0.001821 & 0.001866 & 0.000696 & 0.000005 & 0.000495 & 0.003646 \\
\hline neoC $_{5}$ & 0.000009 & 0.000325 & 0.000399 & 0.000386 & 0.000501 & 0.000501 & 0.000524 & 0.000037 & 0.000325 & 0.000004 & 0.001610 \\
\hline $\mathrm{i}-\mathrm{C}_{5}$ & 0.000001 & 0.000399 & 0.000326 & 0.000314 & 0.000418 & 0.000418 & 0.000440 & 0.000017 & 0.000399 & 0.000000 & 0.001459 \\
\hline$n-C_{5}$ & <empty> & & & & & & & & 0.020997 & & 0.001373 \\
\hline cyc- $C_{5}$ & 0.000445 & <empty> & 0.001444 & 0.001419 & 0.001632 & 0.001632 & 0.001674 & 0.000581 & 0.000000 & 0.000399 & 0.003376 \\
\hline$n-C_{6}$ & 0.000393 & 0.001674 & 0.000009 & 0.000011 & 0.000000 & 0.000000 & <empty> & -0.000700 & 0.010998 & -0.001099 & 0.000297 \\
\hline $\mathrm{MCyc}-\mathrm{C}_{5}$ & 001200 & 0.000581 & 0.000194 & & 0.000266 & 0.000266 & -0.000700 & <empty> & 0.000581 & & 0.001160 \\
\hline benzene & 0.020997 & 0.000000 & 0.001444 & 0.001419 & 0.001632 & 0.001632 & 0.010998 & 0.000581 & <empty> & 0.000399 & 0.002000 \\
\hline cyclohexane & 0.001700 & 0.000399 & 0.000326 & 0.000314 & 0.000418 & 0.000418 & -0.001099 & 0.000017 & 0.000399 & $<$ empty $>$ & 0.001459 \\
\hline$n-C_{7}$ & 0.001373 & 0.003376 & 0.000406 & 0.000420 & 0.000316 & 0.000316 & 0.000297 & 0.001160 & 0.002000 & 0.001459 & <empty > \\
\hline $\mathrm{MCyc}-\mathrm{C}_{6}$ & 0.001300 & 0.001674 & 0.000009 & 0.000011 & 0.000000 & 0.000000 & -0.000300 & 0.000283 & 0.001674 & 0.000440 & 0.000297 \\
\hline toluene & 0.004999 & 0.000528 & 0.000226 & 0.000216 & 0.000304 & 0.000304 & 0.000322 & 0.000001 & 0.000528 & 0.000009 & 0.008000 \\
\hline Cyc-C ${ }_{7}$ & 0.000223 & 0.001297 & 0.000004 & 0.000003 & 0.000019 & 0.000019 & 0.000024 & 0.000142 & 0.001297 & 0.000258 & 0.000490 \\
\hline$n-C_{8}$ & 0.002762 & 0.005411 & 0.001273 & 0.001296 & 0.001108 & 0.001108 & 0.001073 & 0.002457 & 0.004000 & 0.002883 & 0.000241 \\
\hline Cyc- $\mathrm{C}_{8}$ & 0.001060 & 0.002875 & 0.000245 & 0.000255 & 0.000176 & 0.000176 & 0.000162 & 0.000874 & 0.002875 & 0.001135 & 0.000020 \\
\hline $\mathrm{n}-\mathrm{C}_{9}$ & 0.004299 & 0.007486 & 0.002373 & 0.002405 & 0.002146 & 0.002146 & 0.002098 & 0.003917 & 0.007486 & 0.004449 & 0.000818 \\
\hline
\end{tabular}




\begin{tabular}{|c|c|c|c|c|c|c|c|c|c|c|c|}
\hline & I-Сус-C 6 & luene & $y c-C_{7}$ & $\mathrm{n}-\mathrm{C}_{8}$ & Сус-С & $n-C_{9}$ & $\mathrm{n}-\mathrm{C}_{10}$ & m-xylene & p-xylene & $\mathrm{n}-\mathrm{C}_{11}$ & $\mathrm{n}-\mathrm{C}_{12}$ \\
\hline $\mathrm{He}$ & .000000 & 000000 & .000000 & 0.000000 & .000000 & 0.000000 & 0.000000 & 0.000000 & .000000 & 0.000000 & 0.000000 \\
\hline $\mathrm{H}_{2}$ & & .000000 & 0.000000 & 0.000000 & 0.000000 & 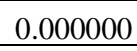 & 0.000000 & 0.000000 & 0.000000 & 0.000000 & 0.375200 \\
\hline $\mathrm{N}_{2}$ & & & & & & & & & & & \\
\hline $\mathrm{CO}_{2}$ & & & 0.108900 & 0.135000 & & & & & & 0.108900 & \\
\hline $\mathrm{CH}_{4}$ & & & & & & & & & & & 0.051799 \\
\hline $\mathrm{C}_{2} \mathrm{H}_{6}$ & & & & & & & & & & 0.030260 & \\
\hline $\mathrm{C}_{3} \mathrm{H}_{8}$ & & & & 0.010 & & & 016630 & & & 0.019476 & 0.022029 \\
\hline $\mathrm{i}-\mathrm{C}_{4}$ & & & & & & & & & & & \\
\hline$n-C_{4}$ & 0.001866 & 0.009999 & 0.001467 & 0.005750 & 0.003124 & 0.007883 & 0.010161 & 0.002083 & 0.002179 & 0.012431 & 0.014506 \\
\hline neo $C_{5}$ & & & & & & & & & & & \\
\hline $\mathrm{i}-\mathrm{C}_{5}$ & 40 & 0.004999 & 0.000258 & 0.002883 & 0.001135 & & 0.006205 & 0.000549 & 0.000598 & 0.008014 & 0.009706 \\
\hline$n-C_{5}$ & & & & & & & & & & & 0.009485 \\
\hline cyc- $-C_{5}$ & & 0.000 & 0.00 & 0.005 & & & & & & & 0.013970 \\
\hline & & & & & & & & & & & 0.006503 \\
\hline & & & & & & & & & & & \\
\hline $2 \mathrm{M}-\mathrm{C}_{5}$ & 0.000000 & 0.000304 & 0.00 & 0.001108 & 0.000176 & 0.002146 & 0.003415 & 0.00 & 0.000016 & 4793 & 0.006126 \\
\hline $3 \mathrm{M}-\mathrm{C}_{5}$ & & & & 0.001108 & & & 0.003415 & & 0.000016 & 4793 & 0.006126 \\
\hline$n-C_{6}$ & -0.000300 & 0.000322 & 0.000024 & 0.001073 & & & & & & & 0.006044 \\
\hline MСус-C ${ }_{5}$ & & & & 0.002457 & & & 0.005575 & & 0.000413 & 0.007297 & 0.008917 \\
\hline & & & & & & & & & & & 0.013970 \\
\hline cyclohexane & & & 0.00 & 0.002883 & 0.001135 & & 0.006205 & & 0.000598 & 0.008014 & 0.009706 \\
\hline $\mathrm{n}-\mathrm{C}_{7}$ & 0.000297 & & & & & & & & & & 0.003673 \\
\hline & & 0.000322 & & 0.00 & & & & & & & 6044 \\
\hline & & & & & & & & & & & \\
\hline Cyc- $\mathrm{C}_{7}$ & & & & & & & & & & & 0.006825 \\
\hline$n-C_{8}$ & 0.001073 & 0.002568 & 0.001418 & <empty> & 0.000402 & 0.000171 & 0.000636 & 0.000919 & 0.008200 & 0.001299 & 0.002036 \\
\hline Сyc- $\mathrm{C}_{8}$ & & & & & & & & & 0.000086 & & 0.004237 \\
\hline$n-C_{9}$ & 0.002098 & 0.004999 & 0.002570 & 0.000171 & 0.001095 & <empty> & 0.000148 & 0.001880 & 0.001791 & 0.000529 & 0.001029 \\
\hline$n-C_{10}$ & 0.003354 & 0.009999 & 0.003944 & 0.000636 & & & & 0.003078 & 0.002964 & 0.000118 & 0.000398 \\
\hline m-xylene & 0.000006 & 0.000416 & 0.000054 & 0.000919 & 0.000106 & 0.001880 & 0.003078 & <empty> & 0.000001 & 0.004392 & 0.005672 \\
\hline p-xylene & & & 0.000071 & 0.008200 & 0.000086 & & 0.002964 & & <empty> & 0.004256 & 0.005518 \\
\hline$n-C_{11}$ & 0.004721 & 0.007487 & 0.005415 & 0.001299 & 0.003140 & 0.000529 & 0.000118 & 0.004392 & 0.004256 & <empty> & 0.000083 \\
\hline$n-C_{12}$ & 0.006044 & 0.009126 & 0.006825 & 0.002036 & 0.004237 & 0.001029 & 0.000398 & 0.005672 & 0.005518 & 0.000083 & <empty> \\
\hline
\end{tabular}


Table S6. The binary interaction parameters for PR and PRSV

\begin{tabular}{|c|c|c|c|c|c|c|c|c|c|c|c|}
\hline & $\mathrm{He}$ & $\mathrm{H}_{2}$ & $\mathrm{~N}_{2}$ & $\mathrm{CO}_{2}$ & $\mathrm{CH}_{4}$ & $\mathrm{C}_{2} \mathrm{H}_{6}$ & $\mathrm{C}_{3} \mathrm{H}_{8}$ & $\mathrm{i}-\mathrm{C}_{4}$ & $\mathrm{n}-\mathrm{C}_{4}$ & neoC $_{5}$ & $\mathrm{i}-\mathrm{C}_{5}$ \\
\hline $\mathrm{He}$ & $<$ empty $>$ & 0.405800 & 0.068498 & 0.796700 & 0.764900 & 1.123200 & 1.064200 & 0.000000 & 0.000000 & 0.000000 & 0.000000 \\
\hline $\mathrm{H}_{2}$ & 0.405800 & <empty> & -0.035999 & 0.120200 & 0.202000 & 0.223100 & 0.214200 & 0.203700 & 0.194100 & 0.292100 & 0.292100 \\
\hline $\mathrm{N}_{2}$ & 0.068498 & -0.035999 & <empty> & -0.019997 & 0.035999 & 0.050000 & 0.079998 & 0.094999 & 0.090000 & 0.100000 & 0.094999 \\
\hline $\mathrm{CO}_{2}$ & 0.796700 & 0.120200 & -0.019997 & <empty> & 0.100000 & 0.129800 & 0.135000 & 0.129800 & 0.129800 & 0.101000 & 0.125000 \\
\hline $\mathrm{CH}_{4}$ & 0.764900 & 0.202000 & 0.035999 & 0.100000 & $<$ empty> & 0.002241 & 0.006829 & 0.013113 & 0.012305 & 0.017131 & 0.017628 \\
\hline $\mathrm{C}_{2} \mathrm{H}_{6}$ & 1.123200 & 0.223100 & 0.050000 & 0.129800 & 0.002241 & <empty> & 0.001258 & 0.004573 & 0.004096 & 0.007088 & 0.007413 \\
\hline $\mathrm{C}_{3} \mathrm{H}_{8}$ & 1.064200 & 0.214200 & 0.079998 & 0.135000 & 0.006829 & 0.001258 & <empty> & 0.001041 & 0.000819 & 0.002392 & 0.002583 \\
\hline $\mathrm{i}-\mathrm{C}_{4}$ & 0.000000 & 0.203700 & 0.094999 & 0.129800 & 0.013113 & 0.004573 & 0.001041 & $<$ empty $>$ & 0.000013 & 0.000278 & 0.000346 \\
\hline$n-C_{4}$ & 0.000000 & 0.194100 & 0.090000 & 0.129800 & 0.012305 & 0.004096 & 0.000819 & 0.000013 & <empty> & 0.000413 & 0.000495 \\
\hline neoC $_{5}$ & 0.000000 & 0.292100 & 0.100000 & 0.101000 & 0.017131 & 0.007088 & 0.002392 & 0.000278 & 0.000413 & <empty> & 0.000004 \\
\hline $\mathrm{i}-\mathrm{C}_{5}$ & 0.000000 & 0.292100 & 0.094999 & 0.125000 & 0.017628 & 0.007413 & 0.002583 & 0.000346 & 0.000495 & 0.000004 & <empty> \\
\hline $\mathrm{n}-\mathrm{C}_{5}$ & 0.000000 & 0.292100 & 0.100000 & 0.125000 & 0.017925 & 0.007609 & 0.002701 & 0.000390 & 0.000547 & 0.000009 & 0.000001 \\
\hline cyc- $-C_{5}$ & 0.000000 & 0.292100 & 0.100000 & 0.101000 & 0.012809 & 0.004393 & 0.000955 & 0.000002 & 0.000005 & 0.000325 & 0.000399 \\
\hline $22 \mathrm{M}-\mathrm{C}_{4}$ & 0.000000 & 0.292100 & 0.100000 & 0.101000 & 0.022613 & 0.010807 & 0.004734 & 0.001343 & 0.001623 & 0.000399 & 0.000326 \\
\hline $23 \mathrm{M}-\mathrm{C}_{4}$ & 0.000000 & 0.292100 & 0.100000 & 0.101000 & 0.022518 & & 0.004690 & 0.001319 & 0.001597 & 0.000386 & 0.000314 \\
\hline $2 \mathrm{M}-\mathrm{C}_{5}$ & 0.000000 & 0.292100 & 0.100000 & 0.101000 & 0.023318 & 0.011303 & 0.005067 & 0.001524 & 0.001821 & 0.000501 & 0.000418 \\
\hline $3 \mathrm{M}-\mathrm{C}_{5}$ & 0.000000 & 0.292100 & 0.100000 & 0.101000 & 0.023318 & 0.011303 & 0.005067 & 0.001524 & 0.001821 & 0.000501 & 0.000418 \\
\hline $\mathrm{n}-\mathrm{C}_{6}$ & 0.000000 & 0.292100 & 0.149000 & 0.125000 & 0.023474 & 0.011414 & 0.005142 & 0.001565 & 0.001866 & 0.000524 & 0.000440 \\
\hline $\mathrm{MCyc}-\mathrm{C}_{5}$ & 0.000000 & 0.292100 & 0.100000 & 0.101000 & 0.018718 & 0.008136 & 0.003020 & 0.000517 & 0.000696 & 0.000037 & 0.000017 \\
\hline benzene & 0.000000 & 0.285100 & 0.159700 & 0.080600 & 0.039999 & 0.019997 & 0.019997 & 0.000002 & 0.000005 & 0.000325 & 0.000399 \\
\hline cyclohexane & 0.000000 & 0.285100 & 0.100000 & 0.090098 & 0.039200 & 0.026100 & 0.014298 & 0.000346 & 0.000495 & 0.000004 & 0.000000 \\
\hline$n-C_{7}$ & 0.000000 & -0.116700 & 0.143900 & 0.119900 & 0.028864 & 0.015324 & 0.007887 & 0.003221 & 0.003646 & 0.001610 & 0.001459 \\
\hline${\mathrm{MCyc}-\mathrm{C}_{6}}_{6}$ & 0.000000 & 0.292100 & 0.106200 & 0.101800 & 0.006500 & 0.012199 & 0.012400 & 0.001565 & 0.001866 & 0.000524 & 0.000440 \\
\hline toluene & 0.000000 & 0.285100 & 0.193200 & 0.093598 & 0.064900 & 0.034400 & 0.030998 & 0.000468 & 0.000639 & 0.000024 & 0.000009 \\
\hline Сyc-C ${ }_{7}$ & 0.000000 & 0.292100 & 0.100000 & 0.101000 & 0.022036 & 0.010403 & 0.004466 & 0.001202 & 0.001467 & 0.000324 & 0.000258 \\
\hline $\mathrm{n}-\mathrm{C}_{8}$ & 0.000000 & 0.292100 & 0.100000 & 0.115000 & 0.034159 & 0.019319 & 0.010850 & 0.005214 & 0.005750 & 0.003093 & 0.002883 \\
\hline Суc-C 8 & 0.000000 & 0.292100 & 0.100000 & 0.101000 & 0.027405 & 0.014248 & 0.007114 & 0.002732 & 0.003124 & 0.001269 & 0.001135 \\
\hline$n-C_{9}$ & 0.000000 & 0.292100 & 0.100000 & 0.101000 & 0.038926 & 0.023017 & 0.013697 & 0.007255 & 0.007883 & 0.004708 & 0.004449 \\
\hline$n-C_{10}$ & 0.000000 & 0.292100 & 0.131600 & 0.118100 & 0.043609 & 0.026730 & 0.016630 & 0.009448 & 0.010161 & 0.006510 & 0.006205 \\
\hline m-xylene & 0.000000 & 0.292100 & 0.216900 & 0.087898 & 0.049100 & 0.029500 & 0.029998 & 0.001765 & 0.002083 & 0.000642 & 0.000549 \\
\hline p-xylene & 0.000000 & 0.292100 & 0.214000 & 0.090000 & 0.050000 & 0.032999 & 0.029998 & 0.001853 & 0.002179 & 0.000696 & 0.000598 \\
\hline$n-C_{11}$ & 0.000000 & 0.292100 & 0.100000 & 0.101000 & 0.047986 & 0.030260 & 0.019476 & 0.011644 & 0.012431 & 0.008359 & 0.008014 \\
\hline$n-C_{12}$ & 0.000000 & 0.356000 & 0.100000 & 0.101000 & 0.051799 & 0.033376 & 0.022029 & 0.013656 & 0.014506 & 0.010084 & 0.009706 \\
\hline
\end{tabular}




\begin{tabular}{|c|c|c|c|c|c|c|c|c|c|c|c|}
\hline & n-C5 & сус-C5 & 22M-C4 & 23M-C4 & 2M-C5 & 3M-C5 & n-C6 & MCус-С5 & benzene & cyclohexane & n-C7 \\
\hline $\mathrm{He}$ & 0.000000 & 0.000000 & 0.000000 & 0.000000 & 0.000000 & 0.000000 & 0.000000 & 0.000000 & 0.000000 & 0.000000 & 0.000000 \\
\hline $\mathrm{H} 2$ & 0.292100 & 0.292100 & 0.292100 & 0.292100 & 0.292100 & 0.292100 & 0.292100 & 0.292100 & 0.285100 & 0.285100 & -0.116700 \\
\hline $\mathrm{N} 2$ & 0.100000 & 0.100000 & 0.100000 & 0.100000 & 0.100000 & 0.100000 & 0.149000 & 0.100000 & 0.159700 & 0.100000 & 0.143900 \\
\hline $\mathrm{CO} 2$ & 0.125000 & 0.101000 & 0.101000 & 0.101000 & 0.101000 & 0.101000 & 0.125000 & 0.101000 & 0.080600 & 0.090098 & 0.119900 \\
\hline $\mathrm{CH} 4$ & 0.017925 & 0.012809 & 0.022613 & 0.022518 & 0.023318 & 0.023318 & 0.023474 & 0.018718 & 0.039999 & 0.039200 & 0.028864 \\
\hline $\mathrm{C} 2 \mathrm{H} 6$ & 0.007609 & 0.004393 & 0.010807 & 0.010740 & 0.011303 & 0.011303 & 0.011414 & 0.008136 & 0.019997 & 0.026100 & 0.015324 \\
\hline $\mathrm{C} 3 \mathrm{H} 8$ & 0.002701 & 0.000955 & 0.004734 & 0.004690 & 0.005067 & 0.005067 & 0.005142 & 0.003020 & 0.019997 & 0.014298 & 0.007887 \\
\hline $\mathrm{i}-\mathrm{C} 4$ & 0.000390 & 0.000002 & 0.001343 & 0.001319 & 0.001524 & 0.001524 & 0.001565 & 0.000517 & 0.000002 & 0.000346 & 0.003221 \\
\hline$n-C 4$ & 0.000547 & 0.000005 & 0.001623 & 0.001597 & 0.001821 & 0.001821 & 0.001866 & 0.000696 & 0.000005 & 0.000495 & 0.003646 \\
\hline neoC5 & 0.000009 & 0.000325 & 0.000399 & 0.000386 & 0.000501 & 0.000501 & 0.000524 & 0.000037 & 0.000325 & 0.000004 & 0.001610 \\
\hline $\mathrm{i}-\mathrm{C} 5$ & 0.000001 & 0.000399 & 0.000326 & 0.000314 & 0.000418 & 0.000418 & 0.000440 & 0.000017 & 0.000399 & 0.000000 & 0.001459 \\
\hline n-C5 & $<$ empty $>$ & 0.000445 & 0.000286 & 0.000275 & 0.000373 & 0.000373 & 0.000393 & 0.001099 & 0.016000 & 0.001300 & 0.001373 \\
\hline сус-C5 & 0.000445 & <empty> & 0.001444 & 0.001419 & 0.001632 & 0.001632 & 0.001674 & 0.000581 & 0.000000 & 0.000399 & 0.003376 \\
\hline 22M-C4 & 0.000286 & 0.001444 & <empty> & 0.000000 & 0.000006 & 0.000006 & 0.000009 & 0.000194 & 0.001444 & 0.000326 & 0.000406 \\
\hline 23M-C4 & 0.000275 & 0.001419 & 0.000000 & <empty> & 0.000007 & 0.000007 & 0.000011 & 0.000185 & 0.001419 & 0.000314 & 0.000420 \\
\hline 2M-C5 & 0.000373 & 0.001632 & 0.000006 & 0.000007 & <empty> & 0.000000 & 0.000000 & 0.000266 & 0.001632 & 0.000418 & 0.000316 \\
\hline $3 \mathrm{M}-\mathrm{C} 5$ & 0.000373 & 0.001632 & 0.000006 & 0.000007 & 0.000000 & $<$ empty $>$ & 0.000000 & 0.000266 & 0.001632 & 0.000418 & 0.000316 \\
\hline n-C6 & 0.000393 & 0.001674 & 0.000009 & 0.000011 & 0.000000 & 0.000000 & <empty> & -0.002300 & 0.007000 & -0.003000 & 0.000297 \\
\hline МСус-C5 & 0.001099 & 0.000581 & 0.000194 & 0.000185 & 0.000266 & 0.000266 & -0.002300 & $<$ empty> & 0.000581 & 0.000017 & 0.001160 \\
\hline benzene & 0.016000 & 0.000000 & 0.001444 & 0.001419 & 0.001632 & 0.001632 & 0.007000 & 0.000581 & <empty> & 0.000399 & -0.002000 \\
\hline cyclohexane & 0.001300 & 0.000399 & 0.000326 & 0.000314 & 0.000418 & 0.000418 & -0.003000 & 0.000017 & 0.000399 & $<$ empty> & 0.001459 \\
\hline n-C7 & 0.001373 & 0.003376 & 0.000406 & 0.000420 & 0.000316 & 0.000316 & 0.000297 & 0.001160 & -0.002000 & 0.001459 & <empty> \\
\hline МСус-С6 & 0.000900 & 0.001674 & 0.000009 & 0.000011 & 0.000000 & 0.000000 & -0.000700 & 0.000283 & 0.001674 & 0.000440 & 0.000297 \\
\hline toluene & 0.000004 & 0.000528 & 0.000226 & 0.000216 & 0.000304 & 0.000304 & 0.000322 & 0.000001 & 0.000528 & 0.000009 & 0.006000 \\
\hline Сус-C7 & 0.000223 & 0.001297 & 0.000004 & 0.000003 & 0.000019 & 0.000019 & 0.000024 & 0.000142 & 0.001297 & 0.000258 & 0.000490 \\
\hline n-C8 & 0.002762 & 0.005411 & 0.001273 & 0.001296 & 0.001108 & 0.001108 & 0.001073 & 0.002457 & 0.003000 & 0.002883 & 0.000241 \\
\hline Сус-С8 & 0.001060 & 0.002875 & 0.000245 & 0.000255 & 0.000176 & 0.000176 & 0.000162 & 0.000874 & 0.002875 & 0.001135 & 0.000020 \\
\hline n-C9 & 0.004299 & 0.007486 & 0.002373 & 0.002405 & 0.002146 & 0.002146 & 0.002098 & 0.003917 & 0.007486 & 0.004449 & 0.000818 \\
\hline n-C10 & 0.006028 & 0.009711 & 0.003700 & 0.003739 & 0.003415 & 0.003415 & 0.003354 & 0.005575 & 0.010000 & 0.010000 & 0.001659 \\
\hline m-xylene & 0.000496 & 0.001880 & 0.000029 & 0.000032 & 0.000009 & 0.000009 & 0.000006 & 0.000372 & 0.001880 & 0.000549 & 0.000219 \\
\hline p-xylene & 0.000544 & 0.001971 & 0.000041 & 0.000045 & 0.000016 & 0.000016 & 0.000012 & 0.000413 & 0.001971 & 0.000598 & 0.000189 \\
\hline n-C11 & 0.007813 & 0.011934 & 0.005128 & 0.005175 & 0.004793 & 0.004793 & 0.004721 & 0.007297 & 0.011934 & 0.008014 & 0.002657 \\
\hline n-C12 & 0.009485 & 0.013970 & 0.006503 & 0.006556 & 0.006126 & 0.006126 & 0.006044 & 0.008917 & 0.013970 & 0.009706 & 0.003673 \\
\hline
\end{tabular}




\begin{tabular}{|c|c|c|c|c|c|c|c|c|c|c|c|}
\hline & I-Сус-C 6 & toluene & $y c-C_{7}$ & $\mathrm{n}-\mathrm{C}_{8}$ & Сус-С & $n-C_{9}$ & $\mathrm{n}-\mathrm{C}_{10}$ & m-xylene & p-xylene & $\mathrm{n}-\mathrm{C}_{11}$ & $\mathrm{n}-\mathrm{C}_{12}$ \\
\hline $\mathrm{He}$ & .000000 & 000000 & .000000 & 0.000000 & .000000 & 0.000000 & 0.000000 & .000000 & .000000 & 0.000000 & 0.000000 \\
\hline $\mathrm{H}_{2}$ & & & 0.292100 & 0.292100 & 0.292100 & مי21020 & 0.292100 & 0.292100 & 0.292100 & 0.292100 & 0.356000 \\
\hline $\mathrm{N}_{2}$ & & & & & & & & & & & \\
\hline $\mathrm{CO}_{2}$ & & 093598 & 0.101000 & 0.115000 & & & & & & 0.101000 & 0.101000 \\
\hline $\mathrm{CH}_{4}$ & & & & & & & & & & & 0.051799 \\
\hline $\mathrm{C}_{2} \mathrm{H}_{6}$ & & & & & & & & & & 0.030260 & \\
\hline $\mathrm{C}_{3} \mathrm{H}_{8}$ & & & & 0.01 & & & 016630 & & 0.029998 & 0.019476 & 0.022029 \\
\hline $\mathrm{i}-\mathrm{C}_{4}$ & & & & & & & & & & & \\
\hline$n-C_{4}$ & 0.001866 & 0.000639 & 0.001467 & 0.005750 & 0.003124 & 0.007883 & 0.010161 & 0.002083 & 0.002179 & 0.012431 & 0.014506 \\
\hline neo $C_{5}$ & & & & & & & & & & & \\
\hline $\mathrm{i}-\mathrm{C}_{5}$ & 000440 & 0.000009 & 0.000258 & 0.002883 & 0.001135 & & 0.006205 & 0.000549 & 0.000598 & 0.008014 & 0.009706 \\
\hline$n-C_{5}$ & & & & & & & & & & & 0.009485 \\
\hline cyc- $-C_{5}$ & & 0.000 & 0.00 & 0.005 & & & & & & & 0.013970 \\
\hline & & & & & & & & & & & 0.006503 \\
\hline $23 \mathrm{M}-\mathrm{C}_{4}$ & & & & & & & & & & & \\
\hline $2 \mathrm{M}-\mathrm{C}_{5}$ & 0.000000 & 0.000304 & 0.00 & 0.001108 & 0.000176 & 0.002146 & 0.003415 & 0009 & 0.000016 & 4793 & 0.006126 \\
\hline $3 \mathrm{M}-\mathrm{C}_{5}$ & & & & & & & 0.003415 & & 0.000016 & 0.00 & 0.006126 \\
\hline$n-C_{6}$ & -0.000700 & 0.000322 & 0.000024 & 0.001073 & & & & & & & 0.006044 \\
\hline MСус-C ${ }_{5}$ & & & & 0.002457 & & & 0.005575 & & 0.000413 & 0.007297 & 0.008917 \\
\hline & & & & & & & & & & & 0.013970 \\
\hline cyclohexane & & & 0.00 & 0.002883 & & & & & 0.000598 & 0.008014 & 0.009706 \\
\hline $\mathrm{n}-\mathrm{C}_{7}$ & 0.000297 & & & & & & & & & & 0.003673 \\
\hline & & 0.000322 & & & & & & & & & 6044 \\
\hline & & & & & & & & & & & \\
\hline Cyc- $\mathrm{C}_{7}$ & & & & & & & & & & & 0.006825 \\
\hline$n-C_{8}$ & 0.001073 & 0.010000 & 0.001418 & <empty> & 0.000402 & 0.000171 & 0.000636 & 0.000919 & 0.006700 & 0.001299 & 0.002036 \\
\hline Сyc- $\mathrm{C}_{8}$ & & & & & & & & & & & 0.004237 \\
\hline$n-C_{9}$ & 0.002098 & 0.004057 & 0.002570 & 0.000171 & 0.001095 & <empty> & 0.000148 & 0.001880 & 0.001791 & 0.000529 & 0.001029 \\
\hline$n-C_{10}$ & 0.003354 & & 0.003944 & 0.000636 & & & & 0.003078 & 0.002964 & 0.000118 & 0.000398 \\
\hline m-xylene & 0.000006 & 0.000416 & 0.000054 & 0.000919 & 0.000106 & 0.001880 & 0.003078 & <empty> & 0.000001 & 0.004392 & 0.005672 \\
\hline p-xylene & & & 0.000071 & 0.006700 & 0.000086 & & 0.002964 & & <empty> & 0.004256 & 0.005518 \\
\hline$n-C_{11}$ & 0.004721 & 0.007487 & 0.005415 & 0.001299 & 0.003140 & 0.000529 & 0.000118 & 0.004392 & 0.004256 & <empty> & 0.000083 \\
\hline$n-C_{12}$ & 0.006044 & 0.009126 & 0.006825 & 0.002036 & 0.004237 & 0.001029 & 0.000398 & 0.005672 & 0.005518 & 0.000083 & <empty> \\
\hline
\end{tabular}


Table S7. The binary interaction parameters for TST

\begin{tabular}{|c|c|c|c|c|c|c|c|c|c|c|c|}
\hline & $\mathrm{He}$ & $\mathrm{H}_{2}$ & $\mathrm{~N}_{2}$ & $\mathrm{CO}_{2}$ & $\mathrm{CH}_{4}$ & $\mathrm{C}_{2} \mathrm{H}_{6}$ & $\mathrm{C}_{3} \mathrm{H}_{8}$ & $\mathrm{i}-\mathrm{C}_{4}$ & $\mathrm{n}-\mathrm{C}_{4}$ & neoC $_{5}$ & $\mathrm{i}-\mathrm{C}_{5}$ \\
\hline $\mathrm{He}$ & $<$ empty> & 0.000000 & 0.000000 & 0.000000 & 0.000000 & 0.000000 & 0.000000 & 0.000000 & 0.000000 & 0.000000 & 0.000000 \\
\hline $\mathrm{H}_{2}$ & 0.000000 & $<$ empty> & 0.000000 & 0.000000 & -0.128464 & -0.180639 & -0.213664 & -0.035994 & -0.152647 & 0.000000 & -0.100000 \\
\hline $\mathrm{N}_{2}$ & 0.000000 & 0.000000 & $<$ empty> & 0.000000 & 0.000000 & 0.000000 & 0.000000 & 0.000000 & 0.000000 & 0.000000 & 0.000000 \\
\hline $\mathrm{CO}_{2}$ & 0.000000 & 0.000000 & 0.000000 & <empty> & 0.000000 & 0.000000 & 0.000000 & 0.000000 & 0.000000 & 0.000000 & 0.000000 \\
\hline $\mathrm{CH}_{4}$ & 0.000000 & -0.128464 & 0.000000 & 0.000000 & <empty> & 0.002241 & 0.006829 & 0.013113 & 0.012305 & 0.017131 & 0.017627 \\
\hline $\mathrm{C}_{2} \mathrm{H}_{6}$ & 0.000000 & -0.180639 & 0.000000 & 0.000000 & 0.002241 & <empty> & 0.001258 & 0.004574 & 0.004096 & 0.007088 & 0.007413 \\
\hline $\mathrm{C}_{3} \mathrm{H}_{8}$ & 0.000000 & -0.213664 & 0.000000 & 0.000000 & 0.006829 & 0.001258 & $<$ empty> & 0.001041 & 0.000819 & 0.002392 & 0.002583 \\
\hline $\mathrm{i}-\mathrm{C}_{4}$ & 0.000000 & -0.035994 & 0.000000 & 0.000000 & 0.013113 & 0.004574 & 0.001041 & <empty> & 0.000013 & 0.000278 & 0.000346 \\
\hline$n-C_{4}$ & 0.000000 & -0.152647 & 0.000000 & 0.000000 & 0.012305 & 0.004096 & 0.000819 & 0.000013 & <empty> & 0.000413 & 0.000495 \\
\hline neoC $_{5}$ & 0.000000 & 0.000000 & 0.000000 & 0.000000 & 0.017131 & 0.007088 & 0.002392 & 0.000278 & 0.000413 & <empty> & 0.000004 \\
\hline $\mathrm{i}-\mathrm{C}_{5}$ & 0.000000 & -0.100000 & 0.000000 & 0.000000 & 0.017627 & 0.007413 & 0.002583 & 0.000346 & 0.000495 & 0.000004 & $<$ empty $>$ \\
\hline$n-C_{5}$ & 0.000000 & -0.218512 & 0.000000 & 0.000000 & 0.017925 & 0.007609 & 0.002700 & 0.000390 & 0.000547 & 0.000009 & 0.000001 \\
\hline cyc- $\mathrm{C}_{5}$ & 0.000000 & 0.000000 & 0.000000 & 0.000000 & 0.012809 & 0.004393 & 0.000955 & 0.000002 & 0.000005 & 0.000325 & 0.000399 \\
\hline $22 \mathrm{M}-\mathrm{C}_{4}$ & 0.000000 & 0.000000 & 0.000000 & 0.000000 & 0.022613 & 0.010807 & 0.004734 & 0.001343 & 0.001623 & 0.000399 & 0.000326 \\
\hline $23 \mathrm{M}-\mathrm{C}_{4}$ & 0.000000 & 0.000000 & 0.000000 & 0.000000 & 0.022518 & 0.010740 & 0.004690 & 0.001319 & 0.001597 & 0.000386 & 0.000314 \\
\hline $2 \mathrm{M}-\mathrm{C}_{5}$ & 0.000000 & 0.000000 & 0.000000 & 0.000000 & 0.023318 & 0.011303 & 0.005067 & 0.001524 & 0.001821 & 0.000501 & 0.000418 \\
\hline $3 \mathrm{M}-\mathrm{C}_{5}$ & 0.000000 & 0.000000 & 0.000000 & 0.000000 & 0.023318 & 0.011303 & 0.005067 & 0.001524 & 0.001821 & 0.000501 & 0.000418 \\
\hline $\mathrm{n}-\mathrm{C}_{6}$ & 0.000000 & 0.000000 & 0.000000 & 0.000000 & 0.023474 & 0.011414 & 0.005142 & 0.001565 & 0.001866 & 0.000524 & 0.000440 \\
\hline MCус-C ${ }_{5}$ & 0.000000 & 0.000000 & 0.000000 & 0.000000 & 0.018718 & 0.008136 & 0.003020 & 0.000517 & 0.000696 & 0.000037 & 0.000017 \\
\hline benzene & 0.000000 & 0.000000 & 0.000000 & 0.000000 & 0.012809 & 0.004393 & 0.000955 & 0.000002 & 0.000005 & 0.000325 & 0.000399 \\
\hline cyclohexane & 0.000000 & 0.000000 & 0.000000 & 0.000000 & 0.017628 & 0.007413 & 0.002583 & 0.000346 & 0.000495 & 0.000004 & 0.000000 \\
\hline $\mathrm{n}-\mathrm{C}_{7}$ & 0.000000 & 0.000000 & 0.000000 & 0.000000 & 0.028864 & 0.015324 & 0.007887 & 0.003221 & 0.003646 & 0.001610 & 0.001459 \\
\hline${\mathrm{MCyc}-\mathrm{C}_{6}}$ & 0.000000 & 0.000000 & 0.000000 & 0.000000 & 0.023474 & 0.011414 & 0.005142 & 0.001565 & 0.001866 & 0.000524 & 0.000440 \\
\hline toluene & 0.000000 & 0.000000 & 0.000000 & 0.000000 & 0.018422 & 0.007938 & 0.002899 & 0.000468 & 0.000639 & 0.000025 & 0.000009 \\
\hline Сyc-C & 0.000000 & 0.000000 & 0.000000 & 0.000000 & 0.022036 & 0.010403 & 0.004466 & 0.001202 & 0.001467 & 0.000324 & 0.000258 \\
\hline $\mathrm{n}-\mathrm{C}_{8}$ & 0.000000 & 0.000000 & 0.000000 & 0.000000 & 0.034159 & 0.019319 & 0.010850 & 0.005214 & 0.005750 & 0.003093 & 0.002883 \\
\hline Сус-C 8 & 0.000000 & 0.000000 & 0.000000 & 0.000000 & 0.027405 & 0.014248 & 0.007114 & 0.002732 & 0.003124 & 0.001269 & 0.001135 \\
\hline$n-C_{9}$ & 0.000000 & 0.000000 & 0.000000 & 0.000000 & 0.038926 & 0.023017 & 0.013697 & 0.007255 & 0.007883 & 0.004708 & 0.004449 \\
\hline$n-C_{10}$ & 0.000000 & 0.000000 & 0.000000 & 0.000000 & 0.043609 & 0.026730 & 0.016630 & 0.009448 & 0.010161 & 0.006510 & 0.006205 \\
\hline m-xylene & 0.000000 & 0.000000 & 0.000000 & 0.000000 & 0.024208 & 0.011935 & 0.005497 & 0.001765 & 0.002083 & 0.000642 & 0.000549 \\
\hline p-xylene & 0.000000 & 0.000000 & 0.000000 & 0.000000 & 0.024523 & 0.012160 & 0.005651 & 0.001853 & 0.002179 & 0.000696 & 0.000598 \\
\hline $\mathrm{n}-\mathrm{C}_{11}$ & 0.000000 & 0.000000 & 0.000000 & 0.000000 & 0.047986 & 0.030260 & 0.019476 & 0.011644 & 0.012431 & 0.008359 & 0.008014 \\
\hline$n-C_{12}$ & 0.000000 & 0.000000 & 0.000000 & 0.000000 & 0.051799 & 0.033376 & 0.022029 & 0.013656 & 0.014506 & 0.010084 & 0.009706 \\
\hline
\end{tabular}




\begin{tabular}{|c|c|c|c|c|c|c|c|c|c|c|c|}
\hline & $\mathrm{n}-\mathrm{C}_{5}$ & cyc- $\mathrm{C}_{5}$ & $22 \mathrm{M}-\mathrm{C}_{4}$ & $23 \mathrm{M}-\mathrm{C}_{4}$ & $2 \mathrm{M}-\mathrm{C}_{5}$ & $3 \mathrm{M}-\mathrm{C}_{5}$ & $n-C_{6}$ & $\mathrm{MCyc}-\mathrm{C}_{5}$ & benzene & cyclohexane & $\mathrm{n}-\mathrm{C}_{7}$ \\
\hline $\mathrm{He}$ & 0.000000 & 0.000000 & 0.000000 & 0.000000 & 0.000000 & 0.000000 & 0.000000 & 0.000000 & 0.000000 & 0.000000 & 0.000000 \\
\hline $\mathrm{H}_{2}$ & -0.218512 & 0.000000 & 0.000000 & 0.000000 & 0.000000 & 0.000000 & 0.000000 & 0.000000 & 0.000000 & 0.000000 & 0.000000 \\
\hline $\mathrm{N} 2$ & 0.000000 & 0.000000 & 0.000000 & 0.000000 & 0.000000 & 0.000000 & 0.000000 & 0.000000 & 0.000000 & 0.000000 & 0.000000 \\
\hline $\mathrm{CO} 2$ & 0.000000 & 0.000000 & 0.000000 & 0.000000 & 0.000000 & 0.000000 & 0.000000 & 0.000000 & 0.000000 & 0.000000 & 0.000000 \\
\hline $\mathrm{CH} 4$ & 0.017925 & 0.012809 & 0.022613 & 0.022518 & 0.023318 & 0.023318 & 0.023474 & 0.018718 & 0.012809 & 0.017628 & 0.028864 \\
\hline $\mathrm{C} 2 \mathrm{H} 6$ & 0.007609 & 0.004393 & 0.010807 & 0.010740 & 0.011303 & 0.011303 & 0.011414 & 0.008136 & 0.004393 & 0.007413 & 0.015324 \\
\hline $\mathrm{C} 3 \mathrm{H} 8$ & 0.002700 & 0.000955 & 0.004734 & 0.004690 & 0.005067 & 0.005067 & 0.005142 & 0.003020 & 0.000955 & 0.002583 & 0.007887 \\
\hline $\mathrm{i}-\mathrm{C} 4$ & 0.000390 & 0.000002 & 0.001343 & 0.001319 & 0.001524 & 0.001524 & 0.001565 & 0.000517 & 0.000002 & 0.000346 & 0.003221 \\
\hline n-C4 & 0.000547 & 0.000005 & 0.001623 & 0.001597 & 0.001821 & 0.001821 & 0.001866 & 0.000696 & 0.000005 & 0.000495 & 0.003646 \\
\hline neoC5 & 0.000009 & 0.000325 & 0.000399 & 0.000386 & 0.000501 & 0.000501 & 0.000524 & 0.000037 & 0.000325 & 0.000004 & 0.001610 \\
\hline $\mathrm{i}-\mathrm{C} 5$ & 0.000001 & 0.000399 & 0.000326 & 0.000314 & 0.000418 & 0.000418 & 0.000440 & 0.000017 & 0.000399 & 0.000000 & 0.001459 \\
\hline $\mathrm{n}-\mathrm{C} 5$ & <empty> & 0.000445 & 0.000286 & 0.000275 & 0.000373 & 0.000373 & 0.000393 & 0.000009 & 0.000445 & 0.000001 & 0.001373 \\
\hline сус-C5 & 0.000445 & <empty> & 0.001444 & 0.001420 & 0.001632 & 0.001632 & 0.001674 & 0.000581 & 0.000000 & 0.000399 & 0.003376 \\
\hline 22M-C4 & 0.000286 & 0.001444 & <empty> & 0.000000 & 0.000006 & 0.000006 & 0.000009 & 0.000194 & 0.001444 & 0.000326 & 0.000406 \\
\hline 23M-C4 & 0.000275 & 0.001420 & 0.000000 & <empty> & 0.000007 & 0.000007 & 0.000011 & 0.000185 & 0.001420 & 0.000314 & 0.000420 \\
\hline 2M-C5 & 0.000373 & 0.001632 & 0.000006 & 0.000007 & <empty> & 0.000000 & 0.000000 & 0.000266 & 0.001632 & 0.000418 & 0.000316 \\
\hline $3 \mathrm{M}-\mathrm{C} 5$ & 0.000373 & 0.001632 & 0.000006 & 0.000007 & 0.000000 & $<$ empty $>$ & 0.000000 & 0.000266 & 0.001632 & 0.000418 & 0.000316 \\
\hline n-C6 & 0.000393 & 0.001674 & 0.000009 & 0.000011 & 0.000000 & 0.000000 & <empty> & 0.000283 & 0.001674 & 0.000440 & 0.000297 \\
\hline MСус-C5 & 0.000009 & 0.000581 & 0.000194 & 0.000185 & 0.000266 & 0.000266 & 0.000283 & <empty> & 0.000581 & 0.000017 & 0.001160 \\
\hline benzene & 0.000445 & 0.000000 & 0.001444 & 0.001420 & 0.001632 & 0.001632 & 0.001674 & 0.000581 & <empty> & 0.000399 & 0.003376 \\
\hline cyclohexane & 0.000001 & 0.000399 & 0.000326 & 0.000314 & 0.000418 & 0.000418 & 0.000440 & 0.000017 & 0.000399 & $<$ empty $>$ & 0.001459 \\
\hline n-C7 & 0.001373 & 0.003376 & 0.000406 & 0.000420 & 0.000316 & 0.000316 & 0.000297 & 0.001160 & 0.003376 & 0.001459 & <empty > \\
\hline MCус-C6 & 0.000393 & 0.001674 & 0.000009 & 0.000011 & 0.000000 & 0.000000 & 0.000000 & 0.000283 & 0.001674 & 0.000440 & 0.000297 \\
\hline toluene & 0.000004 & 0.000528 & 0.000226 & 0.000216 & 0.000304 & 0.000304 & 0.000322 & 0.000001 & 0.000528 & 0.000009 & 0.001238 \\
\hline Сус-C7 & 0.000223 & 0.001297 & 0.000004 & 0.000003 & 0.000019 & 0.000019 & 0.000024 & 0.000142 & 0.001297 & 0.000258 & 0.000490 \\
\hline n-C8 & 0.002762 & 0.005411 & 0.001273 & 0.001296 & 0.001108 & 0.001108 & 0.001073 & 0.002457 & 0.005411 & 0.002883 & 0.000241 \\
\hline Сус-С8 & 0.001060 & 0.002875 & 0.000245 & 0.000255 & 0.000176 & 0.000176 & 0.000162 & 0.000874 & 0.002875 & 0.001135 & 0.000020 \\
\hline n-C9 & 0.004299 & 0.007486 & 0.002373 & 0.002405 & 0.002146 & 0.002146 & 0.002098 & 0.003917 & 0.007486 & 0.004449 & 0.000818 \\
\hline $\mathrm{n}-\mathrm{C} 10$ & 0.006028 & 0.009711 & 0.003700 & 0.003739 & 0.003415 & 0.003415 & 0.003354 & 0.005575 & 0.009711 & 0.006205 & 0.001659 \\
\hline m-xylene & 0.000496 & 0.001880 & 0.000029 & 0.000032 & 0.000009 & 0.000009 & 0.000006 & 0.000372 & 0.001880 & 0.000549 & 0.000219 \\
\hline p-xylene & 0.000544 & 0.001971 & 0.000041 & 0.000045 & 0.000016 & 0.000016 & 0.000012 & 0.000413 & 0.001971 & 0.000598 & 0.000189 \\
\hline n-C11 & 0.007813 & 0.011934 & 0.005128 & 0.005175 & 0.004793 & 0.004793 & 0.004721 & 0.007297 & 0.011934 & 0.008014 & 0.002657 \\
\hline $\mathrm{n}-\mathrm{C} 12$ & 0.009485 & 0.013970 & 0.006503 & 0.006556 & 0.006126 & 0.006126 & 0.006044 & 0.008917 & 0.013970 & 0.009706 & 0.003673 \\
\hline
\end{tabular}




\begin{tabular}{|c|c|c|c|c|c|c|c|c|c|c|c|}
\hline & MCyc-C $_{6}$ & toluene & Сус-C 7 & $\mathrm{n}-\mathrm{C}_{8}$ & Сус-C ${ }_{8}$ & $\mathrm{n}-\mathrm{C}_{9}$ & $\mathrm{n}-C_{10}$ & m-xylene & p-xylene & $\mathrm{n}-\mathrm{C}_{11}$ & $\mathrm{n}-\mathrm{C}_{12}$ \\
\hline $\mathrm{He}$ & 0.000000 & 0.000000 & 0.000000 & 0.000000 & 0.000000 & 0.000000 & 0.000000 & 0.000000 & 0.000000 & 0.000000 & 0.000000 \\
\hline $\mathrm{H}_{2}$ & 0.000000 & 0.000000 & 0.000000 & 0.000000 & 0.000000 & 0.000000 & 0.000000 & 0.000000 & 0.000000 & 0.000000 & 0.000000 \\
\hline $\mathrm{N}_{2}$ & 0.000000 & 0.000000 & 0.000000 & 0.000000 & 0.000000 & 0.000000 & 0.000000 & 0.000000 & 0.000000 & 0.000000 & 0.000000 \\
\hline $\mathrm{CO}_{2}$ & 0.000000 & 0.000000 & 0.000000 & 0.000000 & 0.000000 & 0.000000 & 0.000000 & 0.000000 & 0.000000 & 0.000000 & 0.000000 \\
\hline $\mathrm{CH}_{4}$ & 0.023474 & 0.018422 & 0.022036 & 0.034159 & 0.027405 & 0.038926 & 0.043609 & 0.024208 & 0.024523 & 0.047986 & 0.051799 \\
\hline $\mathrm{C}_{2} \mathrm{H}_{6}$ & 0.011414 & 0.007938 & 0.010403 & 0.019319 & 0.014248 & 0.023017 & 0.026730 & 0.011935 & 0.012160 & 0.030260 & 0.033376 \\
\hline $\mathrm{C}_{3} \mathrm{H}_{8}$ & 0.005142 & 0.002899 & 0.004466 & 0.010850 & 0.007114 & 0.013697 & 0.016630 & 0.005497 & 0.005651 & 0.019476 & 0.022029 \\
\hline $\mathrm{i}-\mathrm{C}_{4}$ & 0.001565 & 0.000468 & 0.001202 & 0.005214 & & & 0.009448 & 0.001765 & 0.001853 & 0.011644 & 0.013656 \\
\hline$n-C_{4}$ & 0.001866 & 0.000639 & 0.001467 & 0.005750 & 0.003124 & 0.007883 & 0.010161 & 0.002083 & 0.002179 & 0.012431 & 0.014506 \\
\hline neoC $_{5}$ & 0.000524 & 0.000025 & 0.000324 & 0.003093 & 0.001269 & 0.004708 & 0.006510 & 0.000642 & 0.000696 & 0.008359 & 0.010084 \\
\hline $\mathrm{i}-\mathrm{C}_{5}$ & 0.000440 & 0.000009 & 0.000258 & 0.002883 & 0.001135 & 0.004449 & 0.006205 & 0.000549 & 0.000598 & 0.008014 & 0.009706 \\
\hline$n-C_{5}$ & 0.000393 & 0.000004 & 0.000223 & 0.002762 & 0.001060 & 0.004299 & 0.006028 & 0.000496 & 0.000544 & 0.007813 & 0.009485 \\
\hline cyc-C ${ }_{5}$ & 0.001674 & 0.000528 & 0.001297 & 0.005411 & 0.002875 & 0.007486 & 0.009711 & 0.001880 & 0.001971 & 0.011934 & 0.013970 \\
\hline $22 \mathrm{M}-\mathrm{C}_{4}$ & 0.000009 & 0.000226 & 0.000004 & 0.001273 & 0.000245 & 0.002373 & 0.003700 & 0.000029 & 0.000041 & 0.005128 & 0.006503 \\
\hline $23 \mathrm{M}-\mathrm{C}_{4}$ & 0.000011 & 0.000216 & 0.000003 & 0.001296 & 0.000255 & 0.002405 & 0.003739 & 0.000032 & 0.000045 & 0.005175 & \\
\hline $2 \mathrm{M}-\mathrm{C}_{5}$ & 0.000000 & 0.000304 & 0.000019 & 0.001108 & 0.000176 & 0.002146 & 0.003415 & 0.000009 & 0.000016 & 0.004793 & 0.006126 \\
\hline $3 \mathrm{M}-\mathrm{C}_{5}$ & 0.000000 & & 0.000019 & 0.001108 & & 0.002146 & 0.003415 & 0.000009 & 0.000016 & 0.004793 & 0.006126 \\
\hline$n-C_{6}$ & 0.000000 & 0.000322 & 0.000024 & 0.001073 & 0.000162 & 0.002098 & 0.003354 & 0.000006 & 0.000012 & 0.004721 & 0.006044 \\
\hline $\mathrm{MCyc} \mathrm{C}_{5}$ & 0.000283 & 0.000001 & 0.000142 & 0.002457 & 0.000874 & 0.003917 & 0.005575 & 0.000372 & 0.000413 & 0.007297 & 0.008917 \\
\hline benzene & 0.001674 & 0.000528 & 0.001297 & 0.005411 & 0.002875 & 0.007486 & 0.009711 & 0.001880 & 0.001971 & 0.011934 & 0.013970 \\
\hline cyclohexane & 0.000440 & 0.000009 & 0.000258 & 0.002883 & 0.001135 & 0.004449 & 0.006205 & 0.000549 & 0.000598 & 0.008014 & 0.009706 \\
\hline$n-C_{7}$ & 0.000297 & 0.001238 & 0.000490 & 0.000241 & 0.000020 & 0.000818 & 0.001659 & 0.000219 & 0.000189 & 0.002657 & 0.003673 \\
\hline $\mathrm{MCyc} \mathrm{C}_{6}$ & <empty> & 0.000322 & 0.000024 & 0.001073 & 0.000162 & 0.002098 & 0.003354 & 0.000006 & 0.000012 & 0.004721 & 0.006044 \\
\hline toluene & 0.000322 & <empty> & 0.000170 & 0.002568 & 0.000941 & 0.004056 & 0.005741 & 0.000416 & 0.000460 & 0.007487 & 0.009126 \\
\hline Сyc-C & 0.000024 & 0.000170 & <empty $>$ & 0.001418 & 0.000311 & 0.002570 & 0.003944 & 0.000054 & 0.000071 & 0.005415 & 0.006825 \\
\hline$n-C_{8}$ & 0.001073 & 0.002568 & 0.001418 & $<$ empty $>$ & 0.000402 & 0.000171 & 0.000636 & 0.000919 & 0.000857 & 0.001299 & 0.002036 \\
\hline Cyc- $\mathrm{C}_{8}$ & 0.000162 & 0.000941 & 0.000311 & 0.000402 & <empty> & 0.001095 & 0.002045 & 0.000106 & 0.000086 & 0.003140 & 0.004237 \\
\hline$n-C_{9}$ & 0.002098 & 0.004056 & 0.002570 & 0.000171 & 0.001095 & <empty> & 0.000148 & 0.001880 & 0.001791 & 0.000529 & 0.001029 \\
\hline$n-C_{10}$ & 0.003354 & 0.005741 & 0.003944 & 0.000636 & 0.002045 & 0.000148 & $<$ empty $>$ & 0.003078 & 0.002964 & 0.000118 & 0.000398 \\
\hline m-xylene & 0.000006 & 0.000416 & 0.000054 & 0.000919 & 0.000106 & 0.001880 & 0.003078 & $<$ empty> & 0.000001 & 0.004392 & 0.005672 \\
\hline p-xylene & 0.000012 & 0.000460 & 0.000071 & 0.000857 & 0.000086 & 0.001791 & 0.002964 & 0.000001 & <empty> & 0.004256 & 0.005518 \\
\hline$n-C_{11}$ & 0.004721 & 0.007487 & 0.005415 & 0.001299 & 0.003140 & 0.000529 & 0.000118 & 0.004392 & 0.004256 & <empty> & 0.000083 \\
\hline$n-C_{12}$ & 0.006044 & 0.009126 & 0.006825 & 0.002036 & 0.004237 & 0.001029 & 0.000398 & 0.005672 & 0.005518 & 0.000083 & <empty> \\
\hline
\end{tabular}


Table S8. The parameter of attractive term for PRSV

\begin{tabular}{|l|l|}
\hline & \multicolumn{1}{|c|}{$k 1$} \\
\hline Helium & 0.270200 \\
\hline Hydrogen & -0.501800 \\
\hline Nitrogen & 0.008860 \\
\hline $\mathrm{CO}_{2}$ & 0.143000 \\
\hline Methane & -0.019270 \\
\hline Ethane & 0.013425 \\
\hline Propane & 0.031628 \\
\hline i-Butane & 0.037810 \\
\hline n-Butane & 0.039507 \\
\hline 22-Mpropane & 0.039609 \\
\hline i-Pentane & 0.044514 \\
\hline n-Pentane & 0.022266 \\
\hline Cyclopentane & 0.064153 \\
\hline 22-Mbutane & -0.071202 \\
\hline 23-Mbutane & 0.069233 \\
\hline 2-Mpentane & 0.072269 \\
\hline 3-Mpentane & 0.070964 \\
\hline n-Hexane & 0.069982 \\
\hline Mcyclopentan & 0.000678 \\
\hline Benzene & 0.046868 \\
\hline Cyclohexane & 0.045120 \\
\hline n-Heptane & 0.072223 \\
\hline Mcyclohexane & 0.077767 \\
\hline Toluene & 0.053640 \\
\hline Cycloheptane & 0.061623 \\
\hline n-Octane & 0.072327 \\
\hline Cyclooctane & 0.201850 \\
\hline n-Nonane & 0.071915 \\
\hline n-Decane & 0.064327 \\
\hline m-Xylene & 0.060100 \\
\hline p-Xylene & 0.032767 \\
\hline n-C11 & 0.067033 \\
\hline n-C12 & 0.064061 \\
\hline
\end{tabular}


Table S9. The parameters for SRK-Twu, PR-Twu, TST

\begin{tabular}{|c|c|c|c|c|c|}
\hline & & \multicolumn{2}{|c|}{$T_{r} \leq 1$} & \multicolumn{2}{|c|}{$T_{r} \geq 1$} \\
\hline & & $\alpha_{i}^{(0)}$ & $\alpha_{i}^{(1)}$ & $\alpha_{i}^{(0)}$ & $\alpha_{i}^{(1)}$ \\
\hline \multirow{3}{*}{ SRK } & $\mathrm{L}$ & 0.544000 & 0.544306 & 0.379919 & 0.0319134 \\
\hline & M & 1.013090 & 0.802404 & 5.673420 & 1.287560 \\
\hline & $\mathrm{N}$ & 0.935995 & 3.108350 & -0.200000 & -8.000000 \\
\hline \multirow{3}{*}{ PR } & $\mathrm{L}$ & 0.272838 & 0.625701 & 0.373949 & 0.023904 \\
\hline & M & 0.924779 & 0.792014 & 4.730200 & 1.246150 \\
\hline & $\mathrm{N}$ & 1.197640 & 2.460220 & -0.200000 & -8.000000 \\
\hline \multirow{3}{*}{$\mathrm{TST}$} & $\mathrm{L}$ & 0.196545 & 0.704001 & 0.358826 & 0.020644 \\
\hline & M & 0.906437 & 0.790407 & 4.234780 & 1.229420 \\
\hline & $\mathrm{N}$ & 1.262510 & 2.130860 & -0.200000 & -8.000000 \\
\hline
\end{tabular}

